\title{
SHAPE OPTIMIZATION OF ONE-CHAMBER MUFFLERS WITH PERFORATED INTRUDING TUBES USING A SIMULATED ANNEALING METHOD
}

\author{
Ying-Chun Chang \\ Department of Mechanical Engineering, Tatung University, Taipei, Taiwan, R.O.C, ycchang@ttu.edu.tw \\ Min-Chie Chiu \\ Department of Automatic Control Engineering, ChungChou Institute of Technology, 6, Lane 2, Sec. 3, Shanchiao Rd., \\ Yuanlin, Changhua 51003, Taiwan, R.O.C \\ Wang-Chuan Liu \\ Department of Mechanical Engineering, Tatung University, Taipei, Taiwan, R.O.C
}

Follow this and additional works at: https://jmstt.ntou.edu.tw/journal

Part of the Engineering Commons

\author{
Recommended Citation \\ Chang, Ying-Chun; Chiu, Min-Chie; and Liu, Wang-Chuan (2010) "SHAPE OPTIMIZATION OF ONE-CHAMBER \\ MUFFLERS WITH PERFORATED INTRUDING TUBES USING A SIMULATED ANNEALING METHOD," Journal of Marine \\ Science and Technology: Vol. 18: Iss. 4, Article 17. \\ DOI: $10.51400 / 2709-6998.1926$ \\ Available at: https://jmstt.ntou.edu.tw/journal/vol18/iss4/17 \\ This Research Article is brought to you for free and open access by Journal of Marine Science and Technology. It has been \\ accepted for inclusion in Journal of Marine Science and Technology by an authorized editor of Journal of Marine Science and \\ Technology.
}


SHAPE OPTIMIZATION OF ONE-CHAMBER MUFFLERS WITH PERFORATED INTRUDING TUBES USING A SIMULATED ANNEALING METHOD

Acknowledgements

The authors acknowledge the financial support of the National Science Council (NSC 97-2221-E-235-001, $\mathrm{ROC})$. 


\title{
SHAPE OPTIMIZATION OF ONE-CHAMBER MUFFLERS WITH PERFORATED INTRUDING TUBES USING A SIMULATED ANNEALING METHOD
}

\author{
Ying-Chun Chang*, Min-Chie Chiu**, and Wang-Chuan Liu*
}

Key words: open-ended perforated tube, decoupled numerical method, space constraints, simulated annealing.

\begin{abstract}
It has been noted that the application of high performance, compact mufflers is the future for modern factories where place is at a premium. However, as research on mufflers equipped with extended tubes has been exhausted, and frankly at the juncture, shown to be inadequate (unsuitable), attention has turned to mufflers conjugated with perforated intruding tubes which can dramatically increase acoustical performance. Therefore, the focus of this paper is not only to analyze the sound transmission loss (STL) of a one-chamber open-ended perforated muffler but also to optimize the best design shape within a limited space.

In this paper, the four-pole system matrix for evaluating the acoustic performance - sound transmission loss (STL) - is derived by using a decoupled numerical method. Additionally, a simulated annealing (SA), a robust scheme used to search for the global optimum by imitating the metal's heating process, has been used during the optimization process. Before dealing with a broadband noise, the STL's maximization with respect to a one-tone noise is introduced for a reliability check on the SA method. Also, an accuracy check on the mathematical model is performed. To appreciate the acoustical ability of the new mufflers, traditional mufflers, including a simple expansion muffler as well as a non-perforated intruding-tube muffler, have been assessed. Results reveal that the maximal STL is precisely located at the desired targeted tone. In addition, the acoustical performance of mufflers conjugated with perforated intruding tubes is found to be superior to the tradi-
\end{abstract}

Paper submitted 07/06/09; revised 08/28/09; accepted 08/29/09. Author for correspondence: Ying-Chun Chang (e-mail: ycchang@ttu.edu.tw).

*Department of Mechanical Engineering, Tatung University, Taipei, Taiwan, R.O.C.

**Department of Automatic Control Engineering, ChungChou Institute of Technology, 6, Lane 2, Sec. 3, Shanchiao Rd., Yuanlin, Changhua 51003, Taiwan, R.O.C. tional mufflers. Consequently, the approach used for the optimal design of the noise elimination proposed in this study is quite effective.

\section{INTRODUCTION}

To overcome the low frequency noise emitted from venting systems, mufflers have been continually used [8]. Research on mufflers was started by Davis et al. in 1954 [4]. Based on the plane wave theory, studies of simple expansion mufflers without perforated holes have been made $[6,10,15]$. To increase a muffler's acoustical performance, the assessment of a new acoustical element - internal perforated plug tubes was discussed by Sullivan and Crocker [19]. On the basis of the coupled differential equations, a series of theoretical and numerical techniques in decoupling the acoustical problems have been proposed [16-18, 20]. In 1981, Jayaraman and Yam [5] developed a method in finding an analytical solution; however, a presumption of the velocity equality within the inner and outer duct, which is not reasonable in the real world, is required. To overcome this drawback, Munjal et al. [12] provided a generalized de-coupling method. Regarding the flowing effect, Peat [14] publicized the numerical decoupling method by finding the eigen value in transfer matrices.

In order to maintain a steady volume-flow-rate in a venting system, a muffler's back pressure within an allowable range is compulsory. Therefore, Wang [21] developed a perforated intruding-tube muffler (a low back-pressure muffler with nonplug tubes inside the cavity) using BEM (boundary element method). However, the need to investigate the optimal muffler design within certain space constraints is rarely seen. In previous work [1-3, 22], the shape optimization of a low backpressure muffler (a one-chamber simple expansion muffler and a one-chamber conjugated muffler with non-perforated intruding tubes) using a GA (genetic algorithm) and gradient methods within a space-constrained situation has been addressed; yet, the acoustical performance of above mufflers is still insufficient. In order to efficiently improve the performance of the noise control device and maintain a steady volumeflow-rate within a space-constrained situation, an optimal 


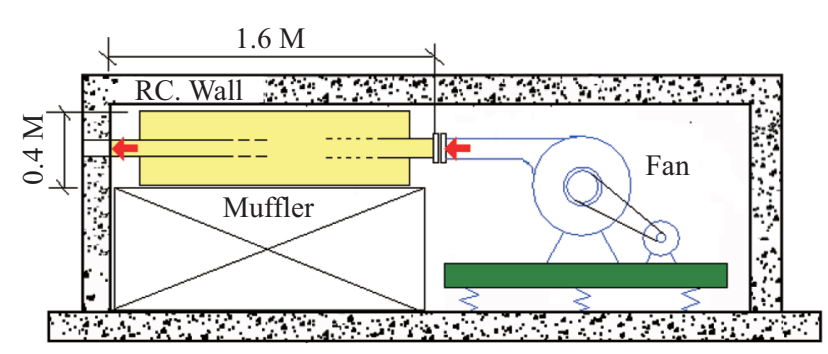

Fig. 1. Noise elimination of a fan noise inside a limited space.

design on a one-chamber muffler equipped with perforated intruding tubes is presented. In this paper, the four-pole system matrix for evaluating the acoustic performance — sound transmission loss (STL) - is derived by using a decoupled numerical method. A SA method patterned after the Darwinian notion of natural selection is applied in this work.

\section{THEORETICAL BACKGROUND}

In this paper, a one-chamber muffler with perforated intruding tubes was adopted for noise elimination in the fan room shown in Fig. 1. Before the acoustical fields of the mufflers are analyzed, the acoustical elements have been distinguished. As shown in Fig. 2, three kinds of muffler components, including three straight tubes, a perforated intruding inlet tube, and a perforated intruding outlet tube, are identified and symbolized as I, II, and III. In addition, the acoustic pressure $\bar{p}$ and acoustic particle velocity $\bar{u}$ within the muffler are depicted in Fig. 3 where the acoustical field is represented by six points.

The muffler system is composed of three kinds of acoustical elements. The individual transfer matrix derivations with respect to three kinds of acoustical mechanisms are described as below.

\section{Transfer Matrix for a Straight Tube}

For a one dimensional wave propagating in a symmetric straight tube shown in Fig. 3, the acoustic pressure and particle velocity are

$$
\begin{gathered}
p(x, t)=\left(k_{1} e^{-j k x /(1+M)}+k_{2} e^{+j k x /(1-M)}\right) e^{j w t} \\
u(x, t)=\left(\frac{k_{1}}{\rho_{o} c_{o}} e^{-j k x /(1+M)}-\frac{k_{2}}{\rho_{o} c_{o}} e^{+j k x /(1-M)}\right) e^{j w t}
\end{gathered}
$$

Considering boundary conditions of pt $0(x=0)$ and pt 1 $(x=L)$, Eqs. (1) and (2) can be rearranged as

$$
\left(\begin{array}{c}
p_{o}(0,0) \\
\rho_{o} c_{o} u_{o}(0,0)
\end{array}\right)=\left[\begin{array}{cc}
1 & 1 \\
1 & -1
\end{array}\right]\left(\begin{array}{l}
k_{1} \\
k_{2}
\end{array}\right)
$$

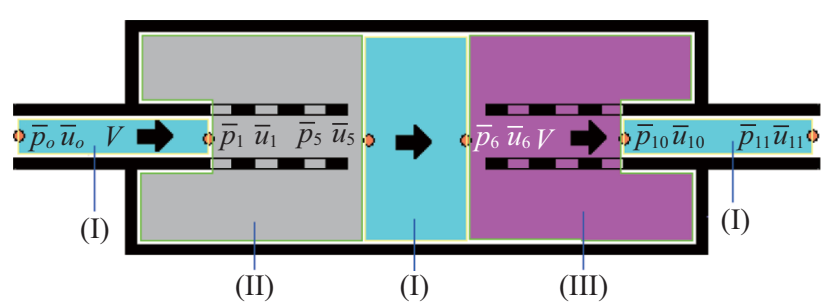

Fig. 2. Acoustical elements in a one-chamber muffler hybridized with perforated intruding tubes.

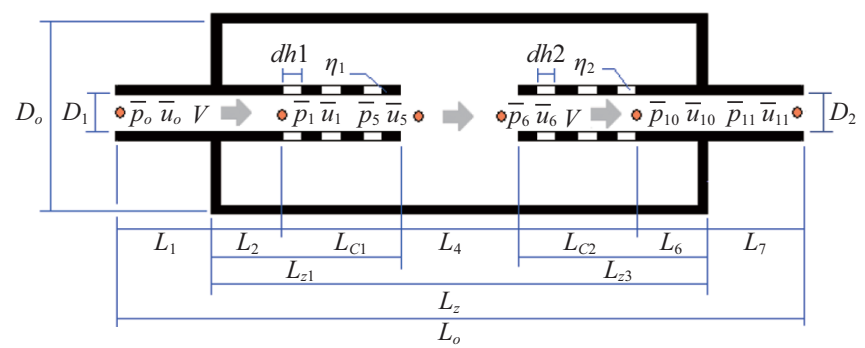

Fig. 3. The outline dimension and acoustical field in a one-chamber muffler hybridized with perforated intruding tubes.

$$
\left(\begin{array}{c}
p_{1}(L, 0) \\
\rho_{o} c_{o} u_{1}(L, 0)
\end{array}\right)=\left[\begin{array}{cc}
e^{-j k^{+} L} & e^{+j k^{-} L} \\
e^{-j k^{+} L} & -e^{+j k^{-} L}
\end{array}\right]\left(\begin{array}{l}
k_{1} \\
k_{2}
\end{array}\right)
$$

where

$$
k^{+}=\frac{k}{1+M_{1}} ; k^{-}=\frac{k}{1-M_{1}} ; L=L_{1}+L_{2}
$$

A combination of (3) and (4) yields

$\left(\begin{array}{c}p_{o}(0,0) \\ \rho_{o} c_{o} u_{o}(0,0)\end{array}\right)$

$=e^{-j \frac{M_{1} k\left(L_{1}+L_{2}\right)}{1-M_{1}^{2}}}\left[\begin{array}{cc}\cos \left(\frac{k\left(L_{1}+L_{2}\right)}{1-M_{1}^{2}}\right) & j \sin \left(\frac{k\left(L_{1}+L_{2}\right)}{1-M_{1}^{2}}\right) \\ j \sin \left(\frac{k\left(L_{1}+L_{2}\right)}{1-M_{1}^{2}}\right) & \cos \left(\frac{k\left(L_{1}+L_{2}\right)}{1-M_{1}^{2}}\right)\end{array}\right]\left(\begin{array}{c}p_{1}(L, 0) \\ \rho_{o} c_{o} u_{1}(L, 0)\end{array}\right)$

or

$$
\left(\begin{array}{c}
\bar{p}_{o} \\
\rho_{o} c_{o} \bar{u}_{o}
\end{array}\right)=e^{-j \frac{M_{1} k\left(L_{1}+L_{2}\right)}{1-M_{1}^{2}}}\left[\begin{array}{ll}
T S 1_{1,1} & T S 1_{1,2} \\
T S 1_{2,1} & T S 1_{2,2}
\end{array}\right]\left(\begin{array}{c}
\bar{p}_{1} \\
\rho_{o} c_{o} \bar{u}_{1}
\end{array}\right)
$$

where

$\bar{p}_{o}=p_{o}(0,0) ; \bar{p}_{1}=p_{1}(L, 0) ; \bar{u}_{o}=u_{o}(0,0) ; \bar{u}_{1}=u_{1}(L, 0)$ 


$$
\begin{aligned}
& T S 1_{1,1}=\cos \left[\frac{k\left(L_{1}+L_{2}\right)}{1-M_{1}^{2}}\right] ; T S 1_{1,2}=j \sin \left[\frac{k\left(L_{1}+L_{2}\right)}{1-M_{1}^{2}}\right] ; \\
& T S 1_{2,1}=j \sin \left[\frac{k\left(L_{1}+L_{2}\right)}{1-M_{1}^{2}}\right] ; T S 1_{2,2}=\cos \left[\frac{k\left(L_{1}+L_{2}\right)}{1-M_{1}^{2}}\right]
\end{aligned}
$$

Similarly, the transfer matrix between pt 5 and pt 6 is

$$
\left(\begin{array}{c}
\bar{p}_{5} \\
\rho_{o} c_{o} \bar{u}_{5}
\end{array}\right)=e^{-j \frac{M_{5} k L_{4}}{1-M_{5}^{2}}}\left[\begin{array}{ll}
T S 2_{1,1} & T S 2_{1,2} \\
T S 2_{2,1} & T S 2_{2,2}
\end{array}\right]\left(\begin{array}{c}
\bar{p}_{6} \\
\rho_{o} c_{o} \bar{u}_{6}
\end{array}\right)
$$

where

$$
\begin{aligned}
& T S 2_{1,1}=\cos \left[\frac{k L_{4}}{1-M_{5}^{2}}\right] ; T S 2_{1,2}=j \sin \left[\frac{k L_{4}}{1-M_{5}^{2}}\right] \\
& T S 2_{2,1}=j \sin \left[\frac{k L_{4}}{1-M_{5}^{2}}\right] ; T S 2_{2,2}=\cos \left[\frac{k L_{4}}{1-M_{5}^{2}}\right]
\end{aligned}
$$

Likewise, the transfer matrix between pt 10 and pt 11 is

$$
\left(\begin{array}{c}
\bar{p}_{10} \\
\rho_{o} c_{o} \bar{u}_{10}
\end{array}\right)=e^{-j \frac{M_{10} k\left(L_{6}+L_{7}\right)}{1-M_{10}^{2}}}\left[\begin{array}{ll}
T S 3_{1,1} & T S 3_{1,2} \\
T S 3_{2,1} & T S 3_{2,2}
\end{array}\right]\left(\begin{array}{c}
\bar{p}_{11} \\
\rho_{o} c_{o} \bar{u}_{11}
\end{array}\right)
$$

where

$$
\begin{aligned}
& T S 3_{1,1}=\cos \left[\frac{k\left(L_{6}+L_{7}\right)}{1-M_{10}^{2}}\right] ; T S 3_{1,2}=j \sin \left[\frac{k\left(L_{6}+L_{7}\right)}{1-M_{10}^{2}}\right] \\
& T S 3_{2,1}=j \sin \left[\frac{k\left(L_{6}+L_{7}\right)}{1-M_{10}^{2}}\right] ; T S 3_{2,2}=\cos \left[\frac{k\left(L_{6}+L_{7}\right)}{1-M_{10}^{2}}\right]
\end{aligned}
$$

\section{Transfer Matrix of a Perforated Intruding Inlet Tube}

To analysis the acoustical mechanism of element (II), a detailed acoustical field represented by five points is depicted in Fig. 4. Based on Sullivan and Crocker's derivation [19], the continuity equations and momentum equations with respect to inner and outer tubes at nodes 1 and 2 are as follows.

\section{Inner tube:}

continuity equation

$$
V_{1} \frac{\partial \rho_{1}}{\partial x}+\rho_{o} \frac{\partial u_{1}}{\partial x}+\frac{4 \rho_{o}}{D_{1}} u+\frac{\partial \rho_{2}}{\partial t}=0
$$

momentum equation:

$$
\rho_{o}\left(\frac{\partial}{\partial t}+V_{1} \frac{\partial}{\partial x}\right) u_{1}+\frac{\partial p_{1}}{\partial x}=0
$$

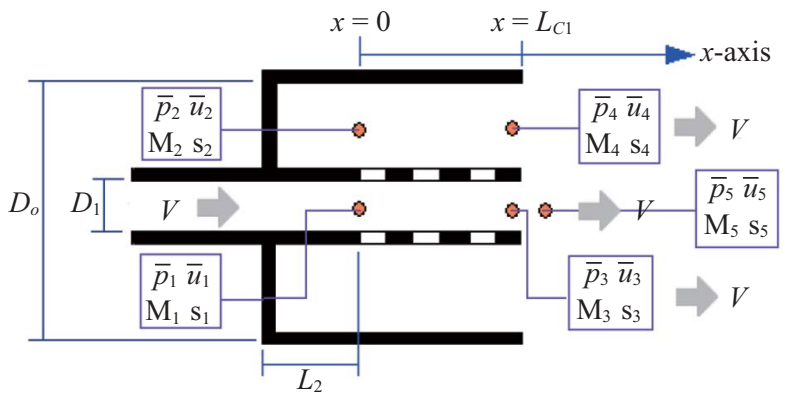

Fig. 4. The acoustical mechanism of a perforated intruding inlet tubes.

\section{Outer tube:}

continuity equation

$$
\begin{gathered}
\rho_{o} \frac{\partial u_{2}}{\partial x}-\frac{4 D_{2} \rho_{o}}{D_{o}^{2}-D_{1}^{2}} u+\frac{\partial \rho_{2}}{\partial t}=0 \\
\rho_{o}\left(\frac{\partial}{\partial t}+V_{2} \frac{\partial}{\partial x}\right) u_{2}+\frac{\partial p_{2}}{\partial x}=0
\end{gathered}
$$

Assuming that the acoustic wave is a harmonic motion

$$
p(x, t)=P(x) \cdot e^{j \omega t}
$$

Under the isentropic processes in ducts, it has

$$
P(x)=\rho(x) \cdot c_{o}^{2}
$$

Assuming that the perforation along the inner tube is uniform (ie. $d \varsigma / d x=0$ ), the acoustic impedance of the perforation $\left(\rho_{o} c_{o} \varsigma\right)$ is

$$
\rho_{o} c_{o} \varsigma=\frac{p_{1}(x)-p_{2}(x)}{u(x)}
$$

where $\varsigma$ is the specific acoustical impedance of the perforated tube. According to the experience formula of $\varsigma$ developed by Sullivan [19] and Rao [16], the empirical formulations for the perforate tube with and without mean flow have been adopted in this study.

For perforates with stationary medium, we have

$$
\varsigma=[0.006+j k(t+0.75 d h)] / \eta
$$

For perforates with grazing flow, we have

$$
\begin{aligned}
\varsigma= & {\left[7.337 \times 10^{-3}(1+72.23 M)\right.} \\
& \left.+j 2.2245 \times 10^{-5}(1+51 t)(1+204 d h) f\right] / \eta
\end{aligned}
$$


where $d h$ is the diameter of the perforated hole on inner tube, $t$ is the thickness of inner perforated tube, and $\eta$ is the porosity of the perforated tube.

The available ranges of above parameters are

$$
\begin{gathered}
0.05 \leqq M \leqq 0.2 ; 0.03 \leqq \eta \leqq 0.1 ; 0.001 \leqq \\
t \leqq 0.003 ; 0.00175 \leqq d h \leqq 0.007
\end{gathered}
$$

By substituting (12)-(14) into (8)-(11), we have

$$
\begin{gathered}
\left\{\frac{d^{2}}{d x^{2}}-\frac{j M_{1}}{1-M_{1}^{2}}\left[\frac{k_{a}^{2}+k^{2}}{k}\right] \frac{d}{d x}+\frac{k_{a}^{2}}{1-M_{1}^{2}}\right\} p_{1} \\
=-\left\{\frac{j M_{1}}{1-M_{1}^{2}}\left[\frac{k_{a}^{2}-k^{2}}{k}\right] \frac{d}{d x}-\frac{k_{a}^{2}-k^{2}}{1-M_{1}^{2}}\right\} p_{2} \\
\left\{\begin{array}{c}
\rho^{2} \\
d x^{2}
\end{array}-\frac{j M_{2}}{1-M_{2}^{2}}\left[\frac{k_{b}^{2}+k^{2}}{k}+V_{1} \frac{\partial}{\partial x}\right) u_{1}+\frac{\partial}{\partial x} p_{1}=0\right. \\
\left.=-\left\{\frac{j M_{2}}{1-M_{2}^{2}}\left[\frac{k_{b}^{2}-k^{2}}{k}\right] \frac{d}{1-M_{2}^{2}}\right\} p_{2}-\frac{k_{b}^{2}-k^{2}}{1-M_{2}^{2}}\right\} p_{1} \\
\rho_{o}\left(\frac{\partial}{\partial t}+V_{2} \frac{\partial}{\partial x}\right) u_{2}+\frac{\partial}{\partial x} p_{2}=0
\end{gathered}
$$

where

$$
\begin{gathered}
k=\frac{\omega}{c} ; M_{j}=\frac{V_{j}}{c} ; j=1,2 ; k_{a}^{2}=k^{2}-j \frac{4 k}{D_{2} \varsigma} \\
k_{b}^{2}=k^{2}-j \frac{4 k D_{2}}{\left(D_{o}^{2}-D_{2}^{2}\right) \varsigma} ; k_{a}^{2}=k^{2}-\frac{i 4 k}{d_{1} \xi}, k_{b}^{2}=k^{2}-\frac{i 4 k d_{1}}{\left(d_{2}^{2}-d_{1}^{2}\right) \xi}
\end{gathered}
$$

Eliminating $u_{1}$ and $u_{2}$ by the differentiation and substitution of (16) yields

$$
\left[\begin{array}{cc}
D^{2}+\alpha_{1} D+\alpha_{2} & \alpha_{3} D+\alpha_{4} \\
\alpha_{5} D+\alpha_{6} & D^{2}+\alpha_{7} D+\alpha_{8}
\end{array}\right]\left[\begin{array}{l}
p_{1} \\
p_{2}
\end{array}\right]=\left[\begin{array}{l}
0 \\
0
\end{array}\right]
$$

where

$$
\begin{gathered}
D=\frac{d}{d x} ; \alpha_{1}=-\frac{j M_{1}}{1-M_{1}^{2}}\left(\frac{k_{a}^{2}+k^{2}}{k}\right) ; \alpha_{2}=\frac{k_{a}^{2}}{1-M_{1}^{2}} ; \\
\alpha_{3}=\frac{j M_{1}}{1-M_{1}^{2}} \cdot\left(\frac{k_{a}^{2}-k^{2}}{k}\right) ; \alpha_{4}=-\left(\frac{k_{a}^{2}-k^{2}}{1-M_{1}^{2}}\right) ;
\end{gathered}
$$

$$
\begin{gathered}
\alpha_{5}=\frac{j M_{2}}{1-M_{2}^{2}}\left(\frac{k_{b}^{2}-k^{2}}{k}\right) ; \alpha_{6}=-\left(\frac{k_{b}^{2}-k^{2}}{1-M_{2}^{2}}\right) ; \\
\alpha_{7}=\frac{j M_{2}}{1-M_{2}^{2}}\left(\frac{k_{b}^{2}+k^{2}}{k}\right) ; \alpha_{8}=\frac{k_{b}^{2}}{1-M_{2}^{2}}
\end{gathered}
$$

Developing (17a), we obtain

$$
\begin{gathered}
p_{1}^{\prime \prime}+\alpha_{1} p_{1}^{\prime}+\alpha_{2} p_{1}+\alpha_{3} p_{2}^{\prime}+\alpha_{4} p_{2}=0 \\
\alpha_{5} p_{1}^{\prime}+\alpha_{6} p_{1}+p_{2}^{\prime \prime}+\alpha_{7} p_{2}^{\prime}+\alpha_{8} p_{2}=0
\end{gathered}
$$

Let $p_{1}^{\prime}=\frac{d p_{1}}{d x}=y_{1}, p_{2}^{\prime}=\frac{d p_{2}}{d x}=y_{2}, p_{1}=y_{3}, p_{2}=y_{4}$

According to (18) and (19), the new matrix between $\left\{y^{\prime}\right\}$ and $\{y\}$ is

$$
\left[\begin{array}{l}
y_{1}^{\prime} \\
y_{2}^{\prime} \\
y_{3}^{\prime} \\
y_{4}^{\prime}
\end{array}\right]=\left[\begin{array}{cccc}
-\alpha_{1} & -\alpha_{3} & -\alpha_{2} & -\alpha_{4} \\
-\alpha_{5} & -\alpha_{7} & -\alpha_{6} & -\alpha_{8} \\
1 & 0 & 0 & 0 \\
0 & 1 & 0 & 0
\end{array}\right]\left[\begin{array}{l}
y_{1} \\
y_{2} \\
y_{3} \\
y_{4}
\end{array}\right]
$$

which can be briefly expressed as

$$
\left\{y^{\prime}\right\}=[\mathrm{N}]\{y\}
$$

Let $\{y\}=[\Omega]\{\Gamma\}$

which is

$$
\left[\begin{array}{c}
d p_{1} / d x \\
d p_{2} / d x \\
p_{1} \\
p_{2}
\end{array}\right]=\left[\begin{array}{llll}
\Omega_{1,1} & \Omega_{1,2} & \Omega_{1,3} & \Omega_{1,4} \\
\Omega_{2,1} & \Omega_{2,2} & \Omega_{2,3} & \Omega_{2,4} \\
\Omega_{3,1} & \Omega_{3,2} & \Omega_{3,3} & \Omega_{3,4} \\
\Omega_{4,1} & \Omega_{4,2} & \Omega_{4,3} & \Omega_{4,4}
\end{array}\right]\left[\begin{array}{c}
\Gamma_{1} \\
\Gamma_{2} \\
\Gamma_{3} \\
\Gamma_{4}
\end{array}\right]
$$

$[\Omega]_{4 \times 4}$ is the model matrix formed by four sets of eigen vectors $\Omega_{4 \times 1}$ of $[\mathrm{N}]_{4 \times 4}$.

Substituting (21) into (20) and then multiplying $[\Omega]^{-1}$ by both sides yields

$$
[\Omega]^{-1}[\Omega]\left\{\Gamma^{\prime}\right\}=[\Omega]^{-1}[\mathrm{~N}][\Omega]\{\Gamma\}
$$

$\operatorname{Set}[\chi]=[\Omega]^{-1}[\mathrm{~N}][\Omega]=\left[\begin{array}{cccc}\beta_{1} & 0 & 0 & 0 \\ 0 & \beta_{2} & 0 & 0 \\ 0 & 0 & \beta_{3} & 0 \\ 0 & 0 & 0 & \beta_{4}\end{array}\right]$ 
where $\beta_{i}$ is the eigen value of $[\mathrm{N}]$

Equation (21) can be thus rewritten as

$$
\left\{\Gamma^{\prime}\right\}=[\chi]\{\Gamma\}
$$

Obviously, Eq. (23) is a decoupled equation. The related solution can then be written as

$$
\Gamma_{i}=C_{i} e^{\beta_{i} x}
$$

Using (9), (11), (21) and (25), the relationship of acoustic pressure and particle velocity is

$$
\left[\begin{array}{c}
p_{1}(x) \\
p_{2}(x) \\
\rho_{o} c_{o} u_{1}(x) \\
\rho_{o} c_{o} u_{2}(x)
\end{array}\right]=\left[\begin{array}{llll}
\mathrm{H}_{1,1} & \mathrm{H}_{1,2} & \mathrm{H}_{1,3} & \mathrm{H}_{1,4} \\
\mathrm{H}_{2,1} & \mathrm{H}_{2,2} & \mathrm{H}_{2,3} & \mathrm{H}_{2,4} \\
\mathrm{H}_{3,1} & \mathrm{H}_{3,2} & \mathrm{H}_{3,3} & \mathrm{H}_{3,4} \\
\mathrm{H}_{4,1} & \mathrm{H}_{4,2} & \mathrm{H}_{4,3} & \mathrm{H}_{4,4}
\end{array}\right]\left[\begin{array}{c}
k_{1} \\
k_{2} \\
k_{3} \\
k_{4}
\end{array}\right]
$$

where

$$
\begin{gathered}
\mathrm{H}_{1, i}=\Omega_{3, i} e^{\beta_{i} x} ; \mathrm{H}_{2, i}=\Omega_{4, i} e^{\beta_{i} x} ; \mathrm{H}_{3, i}=-\Omega_{1, i} \frac{e^{\beta_{i} x}}{j k+M_{1} \beta_{i}} ; \\
\mathrm{H}_{4, i}=-\frac{\Omega_{2, i} e^{\beta_{i} x}}{j k+M_{2} \beta_{i}}
\end{gathered}
$$

Substituting $x=0$ and $x=L_{C 1}$ into (26) yields

$$
\begin{gathered}
{\left[\begin{array}{c}
p_{1}(0) \\
p_{2}(0) \\
\rho_{o} c_{o} u_{1}(0) \\
\rho_{o} c_{o} u_{2}(0)
\end{array}\right]=[\mathrm{H}(0)]\left[\begin{array}{l}
C_{1} \\
C_{2} \\
C_{3} \\
C_{4}
\end{array}\right]} \\
{\left[\begin{array}{c}
p_{1}\left(L_{C}\right) \\
p_{2}\left(L_{C}\right) \\
\rho_{o} c_{o} u_{1}\left(L_{C}\right) \\
\rho_{o} c_{o} u_{2}\left(L_{C}\right)
\end{array}\right]=\left[\mathrm{H}\left(L_{C 1}\right)\right]\left[\begin{array}{c}
C_{1} \\
C_{2} \\
C_{3} \\
C_{4}
\end{array}\right]}
\end{gathered}
$$

Combining (27a) and (27b), the resultant relationship of acoustic pressure and particle velocity between $x=0$ and $x=$ $L_{C 1}$ becomes

$$
\left[\begin{array}{c}
p_{1}(0) \\
p_{2}(0) \\
\rho_{o} c_{o} u_{1}(0) \\
\rho_{o} c_{o} u_{2}(0)
\end{array}\right]=[\mathrm{T}]\left[\begin{array}{c}
p_{1}\left(L_{C}\right) \\
p_{2}\left(L_{C}\right) \\
\rho_{o} c_{o} u_{1}\left(L_{C}\right) \\
\rho_{o} c_{o} u_{2}\left(L_{C}\right)
\end{array}\right]
$$

where

$$
[\mathrm{T}]=[\mathrm{H}(0)]\left[\mathrm{H}\left(L_{C 1}\right)\right]^{-1}=\left[\begin{array}{llll}
T_{1,1} & T_{1,2} & T_{1,3} & T_{1,4} \\
T_{2,1} & T_{2,2} & T_{2,3} & T_{2,4} \\
T_{3,1} & T_{3,2} & T_{3,3} & T_{3,4} \\
T_{4,1} & T_{4,2} & T_{4,3} & T_{4,4}
\end{array}\right]
$$

Let $p_{1}(0)=\bar{p}_{1} ; p_{1}\left(L_{C 1}\right)=\bar{p}_{3} ; u_{1}(0)=\bar{u}_{1} ; u_{1}\left(L_{C 1}\right)=\bar{u}_{3} ;$

$$
p_{2}(0)=\bar{p}_{2} ; p_{2}\left(L_{C 1}\right)=\bar{p}_{4} ; u_{2}(0)=\bar{u}_{2} ; u_{2}\left(L_{C 1}\right)=\bar{u}_{4} ;
$$

Equation (28) can be represented by the new symbols $\bar{p}_{1}, \bar{p}_{2}, \bar{p}_{3}, \bar{p}_{4}, \bar{u}_{1}, \bar{u}_{2}, \bar{u}_{3}$ and $\bar{u}_{4}$

$$
\left[\begin{array}{c}
\bar{p}_{1} \\
\bar{p}_{2} \\
\rho_{o} c_{o} \bar{u}_{1} \\
\rho_{o} c_{o} \bar{u}_{2}
\end{array}\right]=[\mathrm{T}]\left[\begin{array}{c}
\bar{p}_{3} \\
\bar{p}_{4} \\
\rho_{o} c_{o} \bar{u}_{3} \\
\rho_{o} c_{o} \bar{u}_{4}
\end{array}\right]
$$

The equation of mass continuity between point 3 and point 5 with mean flow is expressed in (30)

$$
\begin{aligned}
& c_{o} \rho_{o} S_{3} \bar{u}_{3}+S_{3} M_{3} \bar{p}_{3} \\
& \quad=c_{o} \rho_{o} S_{5} \bar{u}_{5}+c_{o} \rho_{o} S_{4} \bar{u}_{4} \\
& \quad+S_{5} M_{5}\left(\bar{p}_{5}-\frac{p_{o}}{C_{v}} \frac{R K_{e} M_{5} Y_{5}}{p_{o}} \frac{v_{c, 5}-M_{5} p_{c, 5} / Y_{5}}{1-M_{5}^{2}}\right)
\end{aligned}
$$

or

$$
\begin{aligned}
& c_{o} \rho_{o} S_{3} \bar{u}_{3}+S_{3} M_{3} \bar{p}_{3} \\
& \quad=c_{o} \rho_{o} S_{5} \bar{u}_{5}+c_{o} \rho_{o} S_{4} \bar{u}_{4} \\
& \quad+S_{5} M_{5}\left(\bar{p}_{5}-\frac{p_{o}(\gamma-1) K_{e} M_{5} Y_{5}}{p_{o}} \frac{v_{c, 5}-M_{5} p_{c, 5} / Y_{5}}{1-M_{5}^{2}}\right)
\end{aligned}
$$

where

$$
K_{e}=\left[\frac{S_{5}}{S_{3}}-1\right]^{2} ; Y_{5}=\frac{c_{o}}{S_{5}}
$$

A concept of static enthalpy deduced by Munjal [11] is described as

$$
\left[\begin{array}{l}
p_{c, 5} \\
v_{c, 5}
\end{array}\right]=\left[\begin{array}{cc}
1 & M_{5} Y_{5} \\
\frac{M_{5}}{Y_{5}} & 1
\end{array}\right]\left[\begin{array}{c}
\bar{p}_{5} \\
\rho_{o} S_{5} \bar{u}_{5}
\end{array}\right]
$$

Substituting (31) into (30), we have 


$$
\begin{aligned}
& c_{o} \rho_{o} S_{3} \bar{u}_{3}+S_{3} M_{3} \bar{p}_{3} \\
& \quad=c_{o} \rho_{o} S_{5} \bar{u}_{5}\left[1-\frac{(\gamma-1)}{c_{o}} Y_{5} K_{e} S_{5} M_{5}^{2}\right]+\left(M_{5} S_{5} \bar{p}_{5}+c_{o} \rho_{o} \bar{u}_{4} S_{4}\right)
\end{aligned}
$$

or

$$
\begin{aligned}
& c_{o} \rho_{o} S_{3} \bar{u}_{3}+S_{3} M_{3} \bar{p}_{3} \\
& \quad=c_{o} \rho_{o} S_{5} \bar{u}_{5}\left[1-Y_{e}\right]+\left(M_{5} S_{5} \bar{p}_{5}+c_{o} \rho_{o} \bar{u}_{4} S_{4}\right)
\end{aligned}
$$

where

$$
Y_{e}=\frac{(\gamma-1)}{c_{o}} Y_{5} K_{e} S_{5} M_{5}^{2}
$$

The equation of momentum for steady flow is

$$
\begin{aligned}
S_{3} \bar{p}_{3}+2 \rho_{o} S_{3} V_{3} \bar{u}_{3}+S_{3} M_{3}^{2} \bar{p}_{3} & \left(\begin{array}{l}
S_{5} \bar{p}_{5}+2 \rho_{o} S_{5} \bar{u}_{5}+ \\
=-c_{11}\left(\begin{array}{l}
\bar{p}_{5}- \\
\left.S_{5} M_{5}^{2}\left[\begin{array}{l}
v_{c, 5}-M_{5} p_{c, 5} / Y_{5} \\
1-M_{5}^{2}
\end{array}\right]\right)-c_{12} S_{4} \bar{p}_{4}
\end{array}\right)
\end{array}\right.
\end{aligned}
$$

where $c_{11}=-1 ; c_{12}=1$

Substituting (32) into (33), we have

$$
\begin{aligned}
& S_{3}\left(1+M_{3}^{2}\right) \bar{p}_{3}+2 \rho_{o} c_{o} S_{3} M_{3} \bar{u}_{3}+c_{11}\left(S_{5}+S_{5} M_{5}^{2}\right) \bar{p}_{5} \\
& =-c_{11}\left(\frac{2 S_{5}}{c_{o}}-\frac{(\gamma-1) K_{e} M_{5}^{3} Y_{5} S_{5}^{2}}{c_{o}}\right) \rho_{o} c_{o} \bar{u}_{5}-c_{12} S_{4} \bar{p}_{4}
\end{aligned}
$$

The equation of energy conservation for steady flow is

$$
\bar{p}_{3}+\rho_{o} V_{3} \bar{u}_{3}=\bar{p}_{5}+\rho_{o} V_{5} \bar{u}_{5}+K_{e} \rho_{o} V_{5} \bar{u}_{5}
$$

or

$$
\bar{p}_{3}+\rho_{o} V_{3} \bar{u}_{3}=\bar{p}_{5}+\left(1+K_{e}\right) \rho_{o} V_{5} \bar{u}_{5}
$$

With the rigid wall at boundary, we have

$$
\frac{\bar{p}_{2}}{\rho_{o} c_{o} \bar{u}_{2}}=-j \cot \left(k L_{2}\right)
$$

or

$$
\bar{p}_{2} X_{1}=\rho_{o} c_{o} \bar{u}_{2} ; X_{1}=-j \tan \left(k L_{2}\right)
$$

Expanding (29) becomes

$$
\begin{gathered}
\bar{p}_{3}=T_{1,1} \bar{p}_{1}+T_{1,2} \bar{p}_{2}+T_{1,3} \rho_{o} c_{o} \bar{u}_{1}+T_{1,4} \rho_{o} c_{o} \bar{u}_{2} \\
\bar{p}_{1}=T_{2,1} \bar{p}_{1}+T_{2,2} \bar{p}_{2}+T_{2,3} \rho_{o} c_{o} \bar{u}_{1}+T_{2,4} \rho_{o} c_{o} \bar{u}_{2} \\
\rho_{o} c_{o} \bar{u}_{3}=T_{3,1} \bar{p}_{1}+T_{3,2} \bar{p}_{2}+T_{3,3} \rho_{o} c_{o} \bar{u}_{1}+T_{3,4} \rho_{o} c_{o} \bar{u}_{2} \\
\rho_{o} c_{o} \bar{u}_{4}=T_{4,1} \bar{p}_{1}+T_{4,2} \bar{p}_{2}+T_{4,3} \rho_{o} c_{o} \bar{u}_{1}+T_{4,4} \rho_{o} c_{o} \bar{u}_{2}
\end{gathered}
$$

Substituting (36b) into (37a)-(37d) and doing some rearranging, we have

$$
\begin{gathered}
\bar{p}_{3}=T_{1,1} \bar{p}_{1}+\left(T_{1,2}+T_{1,4}\right) \bar{p}_{2}+T_{1,3} \rho_{o} c_{o} \bar{u}_{1} \\
\bar{p}_{4}=T_{2,1} \bar{p}_{1}+\left(T_{2,2}+T_{2,4} X\right) \bar{p}_{2}+T_{2,3} \rho_{o} c_{o} \bar{u}_{1} \\
\rho_{o} c_{o} \bar{u}_{3}=T_{3,1} \bar{p}_{1}+\left(T_{3,2}+T_{3,4} X\right) \bar{p}_{2}+T_{3,3} \rho_{o} c_{o} \bar{u}_{1} \\
\rho_{o} c_{o} \bar{u}_{4}=T_{4,1} \bar{p}_{1}+\left(T_{4,2}+T_{4,4}\right) \bar{p}_{2}+T_{4,3} \rho_{o} c_{o} \bar{u}_{1}
\end{gathered}
$$

By substituting (32b), (34), and (35b) into (38a)-(38d) and eliminating parameters $\bar{p}_{2}, \bar{u}_{2}, \bar{p}_{3}, \bar{u}_{3}, \bar{p}_{4}, \bar{u}_{4}$, the simplified equations become

$$
\bar{p}_{1}=\frac{N_{3} N_{6}-N_{7} N_{2}}{N_{1} N_{6}-N_{5} N_{2}} \bar{p}_{5}+\frac{N_{4} N_{6}-N_{8} N_{2}}{N_{1} N_{6}-N_{5} N_{2}} \rho_{o} c_{o} \bar{u}_{5}
$$

$\rho_{o} c_{o} \bar{u}_{1}=-\frac{N_{3} N_{5}-N_{7} N_{1}}{N_{1} N_{6}-N_{5} N_{2}} \bar{p}_{5}-\frac{N_{4} N_{5}-N_{8} N_{1}}{N_{1} N_{6}-N_{5} N_{2}} \rho_{o} c_{o} \bar{u}_{5}$

where

$N_{1}=-W_{2} W_{5}+W_{1} W_{6} ; N_{2}=W_{3} W_{6}-W_{2} W_{7}$

$N_{3}=-W_{2} W_{8}+W_{6} ; N_{4}=-W_{2} W_{9}+W_{4} W_{6} ;$

$N_{5}=-W_{2} W_{10}+W_{1} W_{11} ; N_{6}=W_{3} W_{11}-W_{2} W_{12} ;$

$N_{7}=W_{11}+W_{2} W_{13} ; N_{8}=W_{4} W_{11}+W_{2} W_{14} ;$

$W_{1}=T_{1,1}+T_{3,1} M_{3}$;

$W_{2}=T_{1,2}+T_{1,4} X_{1}+\left(T_{3,2}+X_{1} T_{3,4}\right) M_{3} ;$

$W_{3}=T_{1,3}+T_{3,3} M_{3} ; W_{4}=\left(1+K_{e}\right) M_{5}$;

$W_{5}=T_{3,1} S_{3}+T_{1,1} S_{3} M_{3}-T_{4,1} S_{4} ;$

$$
\begin{aligned}
W_{6}= & \left(T_{3,2}+X_{1} T_{3,4}\right) S_{3}+\left(T_{1,2}+X_{1} T_{1,4}\right) S_{3} M_{3} \\
& -\left(T_{4,2}+X_{1} T_{4,4}\right) S_{4}
\end{aligned}
$$


$W_{7}=T_{3,3} S_{3}+T_{1,3} S_{3} M_{3}-T_{4,3} S_{4} ; W_{8}=M_{5} S_{5} ;$

$W_{9}=\left(1-Y_{e}+Y_{e} M_{5}^{2}\right) S_{5}$

$W_{10}=T_{1,1}\left(1+M_{3}^{2}\right) S_{3}+2 T_{3,1} S_{3} M_{3}+C_{12} T_{2,1} S_{4}$

$W_{11}=\left(T_{1,2}+X_{1} T_{1,4}\right)\left(1+M_{3}^{2}\right) S_{3}+2\left(T_{3,2}+X_{1} T_{3,4}\right) S_{3} M_{3}$

$$
+C_{12}\left(T_{2,2}+X_{1} T_{2,4}\right) S_{4}
$$

$W_{12}=T_{1,3}\left(1+M_{3}^{2}\right) S_{3}+2 T_{3,3} S_{3} M_{3}+C_{12} T_{2,3} S_{4}$

$W_{13}=C_{11}\left(S_{5}+S_{5} M_{5}^{2}\right)$

$W_{14}=C_{11}\left[2 S_{5} M_{5}-S_{5} M_{5}^{3}(\gamma-1) K_{e}\right]$

Combining (39a) and (39b) into a matrix form yields

$$
\left[\begin{array}{c}
\bar{p}_{1} \\
\rho_{o} c_{o} \bar{u}_{1}
\end{array}\right]=\left[\begin{array}{ll}
\operatorname{TPOE}_{1,1} & T P O E_{1,2} \\
T P O E_{2,1} & T P O E_{2,2}
\end{array}\right]\left[\begin{array}{c}
\bar{p}_{5} \\
\rho_{o} c_{o} \bar{u}_{5}
\end{array}\right]
$$

where

$$
\begin{gathered}
\operatorname{TPOE}_{1,1}=\frac{N_{3} N_{6}-N_{7} N_{2}}{N_{1} N_{6}-N_{5} N_{2}} ; T P O E_{1,2}=\frac{N_{4} N_{6}-N_{8} N_{2}}{N_{1} N_{6}-N_{5} N_{2}} ; \\
\operatorname{TPOE}_{2,1}=\frac{N_{3} N_{5}-N_{7} N_{1}}{N_{1} N_{6}-N_{5} N_{2}} ; T P O E_{2,2}=\frac{N_{4} N_{5}-N_{8} N_{1}}{N_{1} N_{6}-N_{5} N_{2}}
\end{gathered}
$$

\section{Transfer Matrix of a Perforated Intruding Outlet Tube}

Similarly, for a perforated intruding tube shown in Fig. 5, its geometry is symmetrical to the above perforated intruding outlet tube. As derived in (8)-(38), the acoustical pressure and acoustical particle velocity at points 7 and 8 is

$$
\begin{aligned}
& \bar{p}_{7}=\left(T T_{1,1} \bar{p}_{9}+T T_{1,3} X\right) \bar{p}_{9}+T T_{1,2} \bar{p}_{10}+T T_{1,4} \rho_{o} c_{o} \bar{u}_{10} \\
& \bar{p}_{8}=\left(T T_{2,1}+T T_{2,3} X\right) \bar{p}_{9}+T T_{2,2} \bar{p}_{10}+T T_{2,4} \rho_{o} c_{o} \bar{u}_{10} \\
& \rho_{o} c_{o} \bar{u}_{7}=\left(T T_{3,1}+T T_{3,3} X\right) \bar{p}_{9}+T T_{3,2} \bar{p}_{10}+T T_{3,4} \rho_{o} c_{o} \bar{u}_{10} \\
& \rho_{o} c_{o} \bar{u}_{8}=\left(T T_{4,1}+T T_{4,3} X\right) \bar{p}_{9}+T T_{4,2} \bar{p}_{10}+T T_{4,4} \rho_{o} c_{o} \bar{u}_{10}
\end{aligned}
$$

By substituting and eliminating parameters $\left(\bar{p}_{7}, \bar{u}_{7}, \bar{p}_{8}, \bar{u}_{8}\right.$, $\left.\bar{p}_{9}, \bar{u}_{9}\right)$ in (41), the simplified equations become

$\bar{p}_{6}=\frac{N N_{7} N N_{2}-N N_{3} N N_{6}}{N N_{5} N N_{2}-N N_{1} N N_{6}} \bar{p}_{10}+\frac{N N_{8} N N_{2}-N N_{4} N N_{6}}{N N_{5} N N_{2}-N N_{1} N N_{6}} \rho_{o} c_{o} \bar{u}_{10}$

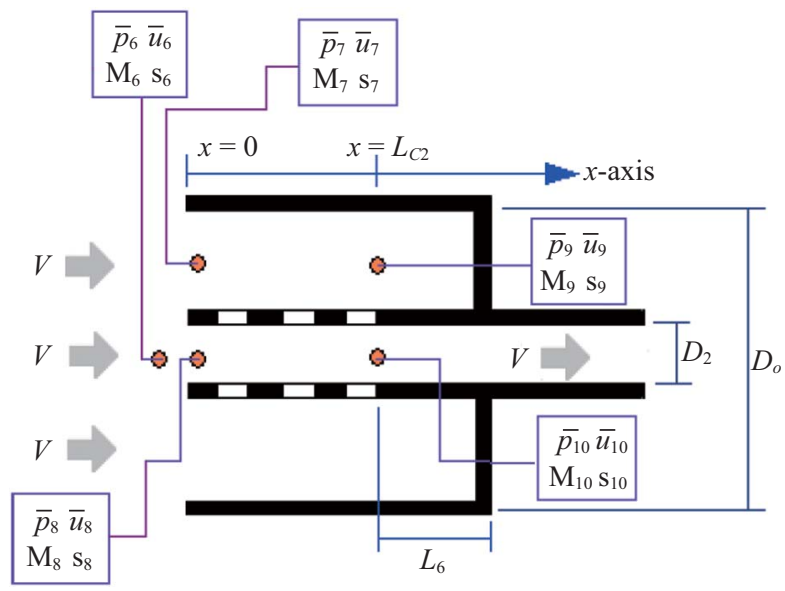

Fig. 5. The acoustical mechanism of a perforated intruding outlet tube.

$\rho_{o} c_{o} \bar{u}_{6}$

$$
=\frac{N N_{7} N N_{1}-N N_{3} N N_{5}}{N N_{6} N N_{1}-N N_{2} N N_{5}} \bar{p}_{10}+\frac{N N_{8} N N_{1}-N N_{4} N N_{5}}{N N_{6} N N_{1}-N N_{2} N N_{5}} \rho_{o} c_{o} \bar{u}_{10}
$$

where

$$
\begin{aligned}
& A_{1}=T T_{1,1}+\left(1+K_{C}\right) M_{8} T T_{3,1} \\
& A_{2}=\left(T T_{1,2}+X_{2} T T_{1,4}\right)+\left(1+K_{C}\right) M_{8}\left(T T_{3,2}+X_{2} T T_{3,4}\right) \\
& A_{3}=T T_{1,3}+\left(1+K_{C}\right) M_{8} T T_{3,3} ; A_{4}=M_{6}
\end{aligned}
$$$$
B_{1}=\left[\left(1-\frac{(\gamma-1) K_{C} M_{8}^{2}}{1-M_{8}^{2}}\right)+\frac{(\gamma-1) K_{C} M_{8}^{4}}{1-M_{8}^{2}}\right] S_{8} T T_{3,1}
$$$$
+M_{8} S_{8} T T_{1,1}+S_{7} T T_{4,1}
$$

$$
\begin{aligned}
B_{2}= & {\left[\left(1-\frac{(\gamma-1) K_{C} M_{8}^{2}}{1-M_{8}^{2}}\right)+\frac{(\gamma-1) K_{C} M_{8}^{4}}{1-M_{8}^{2}}\right]\left[T T_{3,2}+X_{2} T T_{3,4}\right] S_{8} } \\
& +M_{8} S_{8}\left(T T_{1,2}+X_{2} T T_{1,4}\right)+S_{8}\left(T T_{4,2}+X_{2} T T_{4,4}\right)
\end{aligned}
$$

$$
\begin{aligned}
B_{3}= & {\left[\left(1-\frac{(\gamma-1) K_{C} M_{8}^{2}}{1-M_{8}^{2}}\right)+\frac{(\gamma-1) K_{C} M_{8}^{4}}{1-M_{8}^{2}}\right] S_{8} T T_{3,3} } \\
& +M_{8} S_{8} T T_{1,3}+S_{7} T T_{4,3}
\end{aligned}
$$

$B_{4}=S_{6} M_{6} ; B_{5}=S_{6} ;$

$B_{6}=C_{21}\left(S_{8}+S_{8} M_{8}^{2}\right) T T_{1,1}$

$$
+C_{21}\left[2 S_{8} S_{8}-S_{8} M_{8}^{3}(\gamma-1) K_{C}\right] T T_{3,1}+C_{22} S_{7} T T_{2,1}
$$




$$
\begin{aligned}
B_{7}= & C_{21}\left(S_{8}+S_{8} M_{8}^{2}\right)\left(T T_{1,2}+X_{2} T T_{1,4}\right) \\
& +C_{21}\left[2 S_{8} M_{8}-S_{8} M_{8}^{3}(\gamma-1) K_{C}\right]\left(T T_{3,2}+X_{2} T T_{3,4}\right) \\
& +C_{22} S_{7}\left(T T_{2,2}+X_{2} T T_{2,4}\right) ; \\
B_{8}= & C_{21}\left(S_{8}+S_{8} M_{8}^{2}\right) T T_{1,3} \\
& +C_{21}\left[2 S_{8} M_{8}-S_{8} M_{8}^{3}(\gamma-1) K_{C}\right] T T_{3,3}+C_{22} S_{7} T T_{2,3} ; \\
B_{9}= & S_{6}\left(1+M_{6}^{2}\right) ; B_{10}=2 S_{6} M_{6} ; N N_{1}=B_{2}-A_{2} B_{4} ; \\
N N_{2} & =A_{4} B_{2}-A_{2} B_{5} ; N N_{3}=A_{1} B_{2}-A_{2} B_{1} ; N N_{4}=A_{3} B_{2}-A_{2} B_{3} ; \\
N N_{5} & =B_{7}+A_{2} B_{9} ; N N_{6}=A_{4} B_{7}+A_{2} B_{10} ; N N_{7}=A_{1} B_{7}-A_{2} B_{6} ; \\
N N_{8} & =A_{3} B_{7}-A_{2} B_{8}
\end{aligned}
$$

Combining (42a) and (42b) into a matrix form yields

$$
\left[\begin{array}{c}
\bar{p}_{6} \\
\rho_{o} c_{o} \bar{u}_{6}
\end{array}\right]=\left[\begin{array}{ll}
\operatorname{TPOC}_{1,1} & \text { TPOC }_{1,2} \\
\operatorname{TPOC}_{2,1} & \text { TPOC }_{2,2}
\end{array}\right]\left[\begin{array}{c}
\bar{p}_{10} \\
\rho_{o} c_{o} \bar{u}_{10}
\end{array}\right]
$$

where

$$
\begin{aligned}
& \operatorname{TPOC}_{1,1}=\frac{N N_{7} N N_{2}-N N_{3} N N_{6}}{N N_{5} N N_{2}-N N_{1} N N_{6}} ; \\
& \operatorname{TPOC}_{1,2}=\frac{N N_{8} N N_{2}-N N_{4} N N_{6}}{N N_{5} N N_{2}-N N_{1} N N_{6}} ; \\
& \operatorname{TPOC}_{2,1}=\frac{N N_{7} N N_{1}-N N_{3} N N_{5}}{N N_{6} N N_{1}-N N_{2} N N_{5}} ; \\
& \operatorname{TPOC}_{2,2}=\frac{N N_{8} N N_{1}-N N_{4} N N_{5}}{N N_{6} N N_{1}-N N_{2} N N_{5}}
\end{aligned}
$$

\section{Sound Transmission Loss}

For combining (5), (6), (7), (40), and (43), the total transfer matrix assembled by multiplication is

$$
\begin{aligned}
& \left(\begin{array}{c}
\bar{p}_{o} \\
\rho_{o} c_{o} \bar{u}_{o}
\end{array}\right) \\
& =e^{-j k\left[\frac{M_{1}\left(L_{1}+L_{2}\right)}{\left.1-M_{1}^{2}+\frac{M_{5} k L_{4}}{1-M_{5}^{2}}+\frac{M_{10}\left(L_{6}+L_{7}\right)}{1-M_{10}^{2}}\right]}\left[\begin{array}{ll}
T S 1_{1,1} & T S 1_{1,2} \\
T S 1_{2,1} & T S 1_{2,2}
\end{array}\right]\right.} \\
& {\left[\begin{array}{ll}
T P O E_{1,1} & T P O E_{1,2} \\
T P O E_{2,1} & T P O E_{2,2}
\end{array}\right]\left[\begin{array}{ll}
T S 2_{1,1} & T S 2_{1,2} \\
T S 2_{2,1} & T S 2_{2,2}
\end{array}\right]}
\end{aligned}
$$

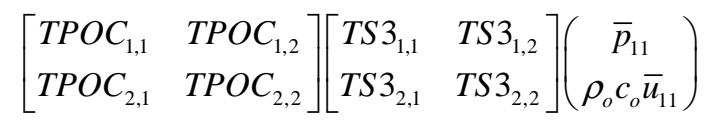

A simplified form in the matrix is expressed as

$$
\left(\begin{array}{c}
\bar{p}_{o} \\
\rho_{o} c_{o} \bar{u}_{o}
\end{array}\right)=\left[\begin{array}{cc}
T_{11}^{*} & T_{12}^{*} \\
T_{21}^{*} & T_{22}^{*}
\end{array}\right]\left(\begin{array}{c}
\bar{p}_{11} \\
\rho_{o} c_{o} \bar{u}_{11}
\end{array}\right)
$$

Under the assumption of a fixed thickness of the tubes $\left(t_{1}=\right.$ $\left.t_{2}=0.001 \mathrm{~m}\right)$ and the symmetric design $\left(L_{1}=L_{7}=\left(L_{o}-L_{z}\right) / 2\right.$; $\left.L_{z 1}=L_{z 3}=\left(L_{z}-L_{4}\right) / 2\right)$ ), the sound transmission loss (STL) of a muffler is defined as [10]

$$
\begin{aligned}
& S T L\left(Q, f, R T_{1}, R T_{2}, R T_{3}, R T_{4}, R T_{5}, R T_{6}, R T_{7}, R T_{8}, R T_{9}, R T_{10}\right) \\
& \quad=\log \left(\frac{\left|T_{11}^{*}+T_{12}^{*}+T_{21}^{*}+T_{22}^{*}\right|}{2}\right)+10 \log \left(\frac{S_{1}}{S_{11}}\right)
\end{aligned}
$$

where

$R T_{1}=L_{z} / L_{o} ; R T_{2}=L_{4} / L_{z} ; R T_{3}=L_{C 1} / L_{z 1} ; R T_{4}=L_{C 2} / L_{z 2} ;$

$R T_{5}=D_{1} / D_{o} ; R T_{6}=D_{2} / D_{o} ; R T_{7}=d h_{1} ; R T_{8}=\eta_{1} ; R T_{9}=d h_{2} ;$

$R T_{10}=\eta_{2} ; L_{o}=L_{1}+L_{z}+L_{7} ; L_{z}=L_{z 1}+L_{4}+L_{z 3} ;$

$L_{z 1}=L_{2}+L_{C 1} ; L_{z 3}=L_{6}+L_{C 2} ; L_{1}=L_{7}=\left(L_{o}-L_{z}\right) / 2 ;$

$\left.L_{z 1}=L_{z 3}=\left(L_{z}-L_{4}\right) / 2\right)$

\section{Overall Sound Power Level}

The silenced octave sound power level emitted from a silencer's outlet is

$$
S W L_{i}=S W L O_{i}-S T L_{i}
$$

where (1) $S W L O_{i}$ is the original $S W L$ at the inlet of a muffler (or pipe outlet), and $i$ is the index of the octave band frequency.

(2) $S T L_{i}$ is the muffler's $S T L$ with respect to the relative octave band frequency.

(3) $S W L_{i}$ is the silenced $S W L$ at the outlet of a muffler with respect to the relative octave band frequency.

Finally, the overall $S W L_{T}$ silenced by a muffler at the outlet is

$$
\begin{aligned}
& S W L_{T}=10 * \log \left\{\sum_{i=1}^{5} 10^{S W L_{i} / 10}\right\} \\
& =10 * \log \left\{\begin{array}{ccc}
{[S W L O(f=125)-} & {[S W L(f=250)-} \\
10^{S L L(f=125)]}+10^{S T L(f=250)] / 10} & \\
{[S W L O(f=500)-} & {[S W L O(f=1000)-} & {[S W L O(f=2000)-} \\
+10^{S L L(f=500)] / 10}+10^{S L L(f=1000)] / 10}+10^{S T L(f=2000)] / 10}
\end{array}\right\}
\end{aligned}
$$



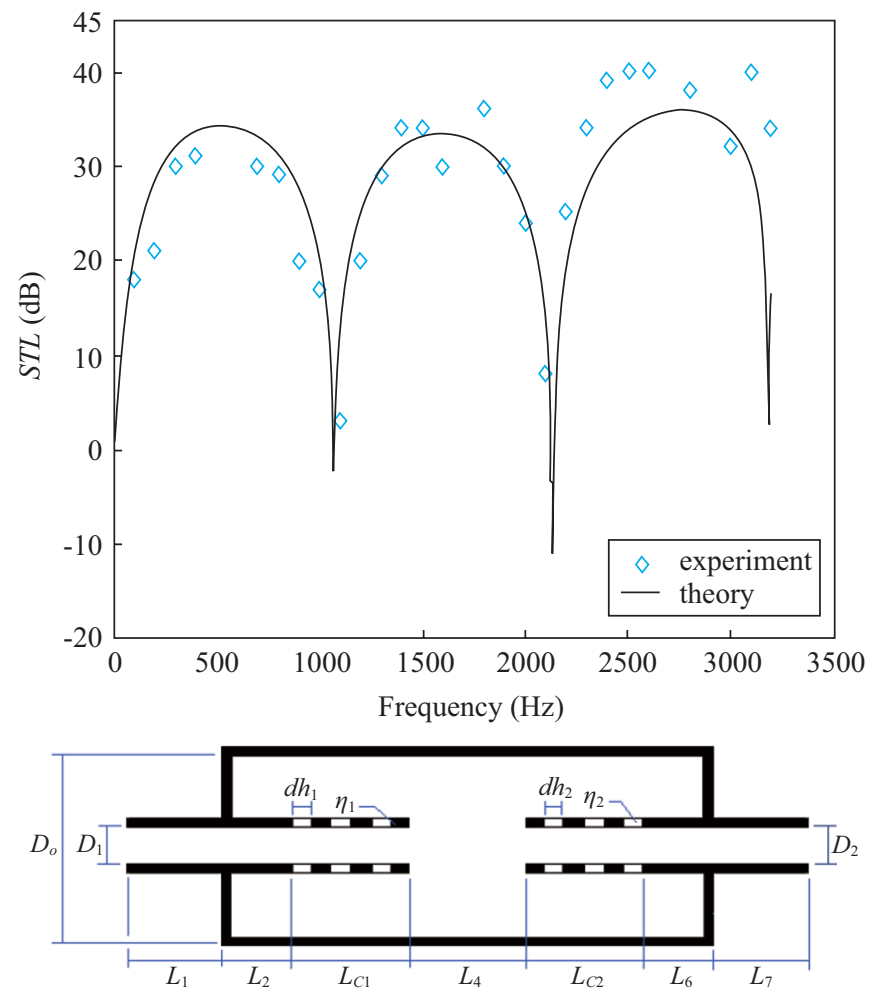

Fig. 6. Performance of a one-chamber muffler equipped with perforated intruding tubes $\left[D_{1}=0.018(\mathrm{~m}), D_{2}=0.018(\mathrm{~m}), D_{o}=0.118(\mathrm{~m})\right.$, $L_{z}=0.16, L_{4}=0.08, L_{2}=L_{6}=0.0, L_{C 1}=L_{C 2}=0.04, L_{1}=L_{7}=0.04$, $t_{1}=t_{2}=0.001(\mathrm{~m}), d h_{1}=d h_{2}=0.003(\mathrm{~m}), \eta_{1}=\eta_{2}=0.03375, M_{1}=$ 0.0] [Experimental data is from Wang et al. [21]].

\section{Objective Function}

By using the formulas of (46) and (48), the objective function used in the SA optimization was established.

1) STL Maximization for a One-Tone (f) Noise

$O B J_{1}=\left(Q, f, R T_{1}, R T_{2}, R T_{3}, R T_{4}, R T_{5}, R T_{6}, R T_{7}, R T_{8}, R T_{9}, R T_{10}\right)$

\section{2) SWL Minimization for a Broadband Noise}

To minimize the overall $S W L_{T}$, the objective function is

$$
\mathrm{OBJ}_{2}
$$$$
=S W L_{T}\left(Q, f, R T_{1}, R T_{2}, R T_{3}, R T_{4}, R T_{5}, R T_{6}, R T_{7}, R T_{8}, R T_{9}, R T_{10}\right)
$$

The related ranges of parameters are

$f=250(\mathrm{~Hz}), \mathrm{Q}=0.05\left(\mathrm{~m}^{3} / \mathrm{s}\right) ; D_{\mathrm{o}}=0.4(\mathrm{~m}), L_{\mathrm{o}}=1.6(\mathrm{~m}) ;$

$R T_{1}$ : [0.5, 0.9]; $R T_{2}:[0.1,0.5] ; R T_{3}:[0.2,0.8] ; R T_{4}:[0.2,0.8]$;
Table 1. Unsilenced $S W L$ of a fan inside a duct outlet.

\begin{tabular}{|c|c|c|c|c|c|c|}
\hline Frequency - Hz & 125 & 250 & 500 & 1000 & 2000 & 4000 \\
\hline$S W L O-\mathrm{dB}$ & 115 & 120 & 116 & 102 & 96 & 88 \\
\hline
\end{tabular}

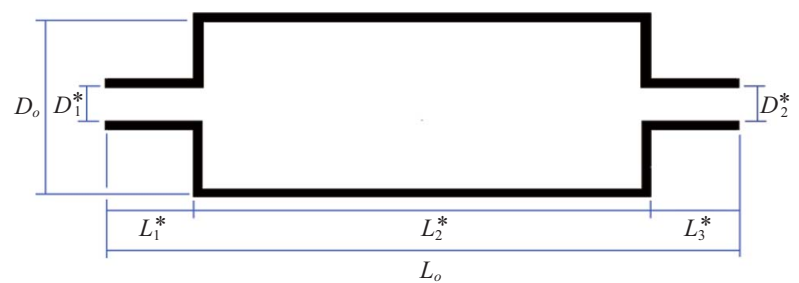

(a)

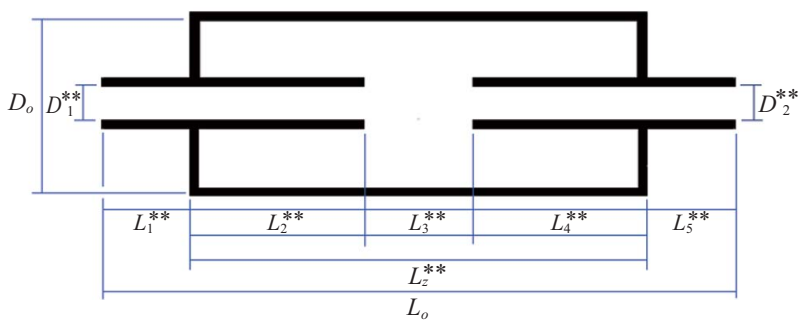

(b)

Fig. 7. Two kinds of low back-pressure mufflers [(a) a one-chamber simple expansion muffler; (b) a one-chamber muffler hybridized with nonperforated intruding tubes].

$R T_{5}:[0.2,0.8] ; R T_{6}:[0.2,0.8] ; R T_{7}:[0.00175,0.007]$;

$R T_{8}:[0.03,0.1] ; R T_{9}:[0.00175,0.007] ; R T_{10}:[0.03,0.1]$

\section{MODEL CHECK}

Before performing the SA optimal simulation on mufflers, an accuracy check of the mathematical model on a one-chamber muffler with perforated intruding tubes is performed by Wang et al. [21]. As indicated in Fig. 6, the accuracy comparisons between theoretical data and analytical data are in agreement. Therefore, the model of a one-chamber muffler with perforated intruding tubes is acceptable and adopted in the following optimization process.

\section{CASE STUDIES}

In this paper, the noise reduction of a space-constrained fan room is exemplified and shown in Fig. 1. The sound power level (SWL) inside the fan's outlet is shown in Table 1 where the overall SWL reaches $122.4 \mathrm{~dB}$. To depress the huge venting noise emitted from the fan's outlet, a one-chamber muffler hybridized with perforated intruding tubes is considered. To obtain the best acoustical performance within a fixed space, numerical assessments linked to a SA optimizer are applied. Before the minimization of a broadband noise is executed, a reliability check of the SA method by maximization of the STL at a targeted one tone $(250 \mathrm{~Hz})$ has been carried out. As indicated in Fig. 7, to appreciate the acoustic performance, 


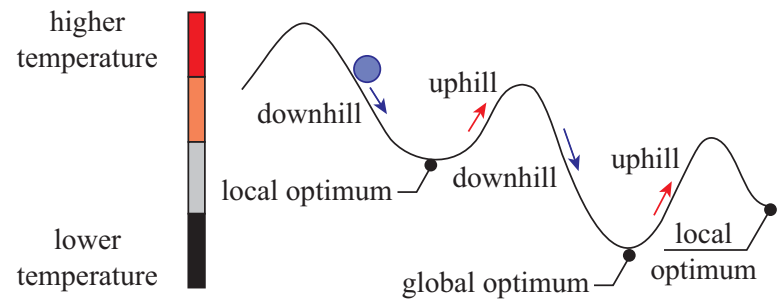

Fig. 8. SA algorithm from a physical viewpoint.

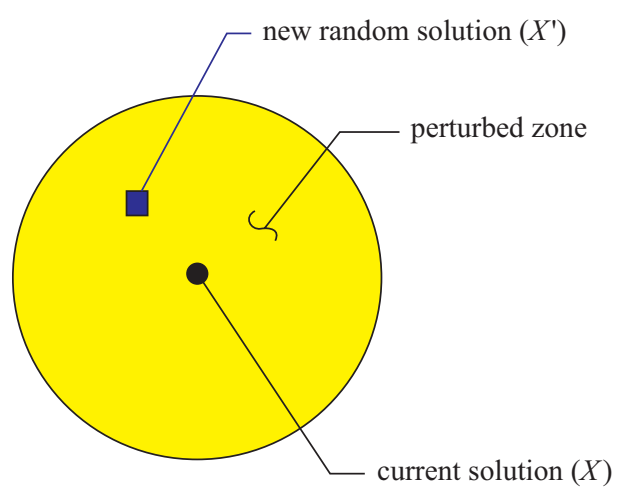

Fig. 9. New random solution in a perturbed zone.

two kinds of low back-pressure mufflers (one, a simple expansion muffler; and the other, a muffler hybridized with nonperforated intruding tubes) are accessed and optimized. As shown in Figs. 1 and 2, the available space for a muffler is 0.4 $\mathrm{m}$ in width, $0.4 \mathrm{~m}$ in height, and $1.6 \mathrm{~m}$ in length. The flow rate $(Q)$ and thickness of a perforated tube $(\mathrm{t})$ are preset at 0.05 $\left(\mathrm{m}^{3} / \mathrm{s}\right)$ and $0.001(\mathrm{~m})$, respectively. The corresponding $O B J$ functions, space constraints, and the ranges of design parameters are summarized in (49)-(51).

\section{SIMULATED ANNEALING ALGORITHM}

The basic concept behind simulated annealing (SA) was first introduced by Metropolis et al. [9] and developed by Kirkpatrick et al. [7]. The simulated annealing (SA) algorithm is a local search process which imitates the softening process (annealing) of metal. In the physical system, annealing is the process of heating and keeping a metal at a stabilized temperature while cooling it slowly. Slow cooling allows the particles to keep their state close to the minimal energy state. In this state, the particles have a more homogeneous crystalline structure. Conversely, a fast cooling rate results in a higher distorted energy that is stored inside the imperfect lattice.

The algorithm starts by generating a random initial solution. The scheme of SA is a variation of the hill-climbing algorithm. As indicated in Fig. 8, all downhill movements for improvement are accepted for the decrement of the system's energy. Simultaneously, SA also allows movement resulting in solutions that are worse (uphill moves) than the current solution. This is done in order to escape from the local optimum.

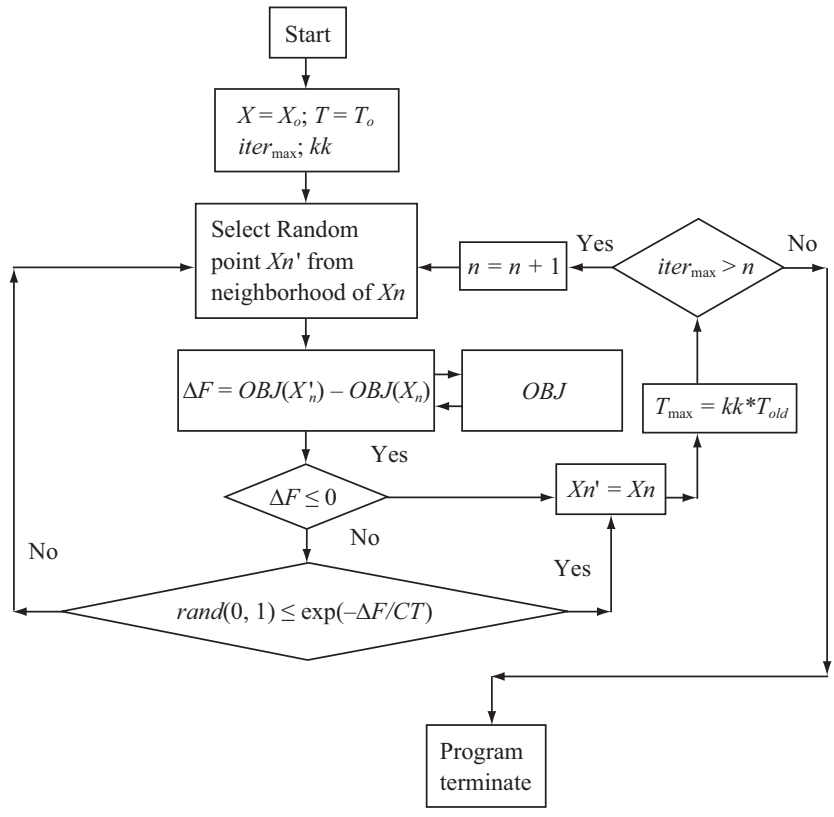

Fig. 10. Flow diagram of a SA optimization.

As indicated in Fig. 9, to imitate the evolution of the SA algorithm, a new random solution $\left(X^{\prime}\right)$ is chosen from the neighborhood of the current solution $(X)$. If the change in the objective function (or energy) is negative, ie., $\Delta F \leq 0$, a new solution will be acknowledged as the new current solution with the transition property $p b\left(X^{\prime}\right)$ of 1 ; if it is not negative, then the new transition property $\left(p b\left(X^{\prime}\right)\right)$ varying from $0 \sim 1$ will be calculated by the Boltzmann's factor $\left(p b\left(X^{\prime}\right)=\right.$ $\exp (\Delta F / C T))$ as shown in (52).

$$
\begin{gathered}
p b\left(X^{\prime}\right)=\left(\begin{array}{c}
1, \Delta F \leq 0 \\
\exp \left(\frac{-\Delta F}{C T}\right), \Delta f>0
\end{array}\right. \\
\Delta F=O B J\left(X^{\prime}\right)-O B J(X)
\end{gathered}
$$

where the $C$ and $T$ are the Boltzmann constant and the current temperature. Additionally, compared to the new random probability of $\operatorname{rand}(0,1)$, if the transition property $\left(p b\left(X^{\prime}\right)\right)$ is greater than a random number of $\operatorname{rand}(0,1)$, the new uphill solution, which results in a higher energy condition, will then be accepted; otherwise, it is rejected. The uphill solution at a higher temperature will therefore have a better chance of escaping from the local optimum. The algorithm repeats the perturbation of the current solution and the measurement of the change in the objective function. As indicated in Fig. 10, each successful substitution of the new current solution will lead to the decay of the current temperature as

$$
T_{\text {new }}=k k * T_{\text {old }}
$$


Table 2. Optimal $S T L$ for a one-chamber muffler with perforated intruding tubes at various $k k$ and iter $_{\text {max }}$ (targeted tone of $250 \mathrm{~Hz}$ ).

\begin{tabular}{|c|c|c|c|c|c|c|c|c|}
\hline \multirow{6}{*}{$\begin{array}{c}\text { Item } \\
1\end{array}$} & \multicolumn{2}{|c|}{$S A$ parameters } & \multirow{2}{*}{\multicolumn{6}{|c|}{ Results }} \\
\hline & \multirow{5}{*}{\begin{tabular}{|c|}
$k k$ \\
0.90
\end{tabular}} & \multirow{5}{*}{$\frac{\text { iter }_{\max }}{200}$} & & & & & & \\
\hline & & & $R T 1$ & $R T 2$ & $R T 3$ & $R T 4$ & $R T 5$ & \multirow{4}{*}{\begin{tabular}{|c|}
$S T L(\mathrm{~dB}$ \\
44.3
\end{tabular}} \\
\hline & & & 0.7659 & 0.3659 & 0.5989 & 0.5989 & 0.59989 & \\
\hline & & & $R T 6$ & $R T 7$ & $R T 8$ & $R T 9$ & $R T 10$ & \\
\hline & & & 0.5989 & 0.005241 & 0.07654 & 0.005241 & 0.07654 & \\
\hline \multirow[t]{4}{*}{2} & \multirow[t]{4}{*}{0.93} & \multirow[t]{4}{*}{200} & $R T 1$ & $R T 2$ & $R T 3$ & $R T 4$ & $R T 5$ & $S T L(\mathrm{~dB})$ \\
\hline & & & 0.8428 & 0.4428 & 0.7141 & 0.7141 & 0.7141 & \multirow[t]{3}{*}{55.4} \\
\hline & & & $R T 6$ & $R T 7$ & $R T 8$ & $R T 9$ & $R T 10$ & \\
\hline & & & 0.7141 & 0.006249 & 0.08998 & 0.006249 & 0.08998 & \\
\hline \multirow[t]{4}{*}{3} & \multirow[t]{4}{*}{$\underline{0.96}$} & \multirow{4}{*}{200} & $R T 1$ & $R T 2$ & $R T 3$ & $R T 4$ & $R T 5$ & $S T L(\mathrm{~dB})$ \\
\hline & & & 0.8664 & 0.4664 & 0.7496 & 0.7496 & 0.7496 & \multirow[t]{3}{*}{63.7} \\
\hline & & & $R T 6$ & $R T 7$ & $R T 8$ & $R T 9$ & $R T 10$ & \\
\hline & & & 0.7496 & 0.006559 & 0.09412 & 0.006559 & 0.09412 & \\
\hline \multirow[t]{4}{*}{4} & \multirow[t]{4}{*}{0.99} & \multirow[t]{4}{*}{200} & $R T 1$ & $R T 2$ & $R T 3$ & $R T 4$ & $R T 5$ & $S T L(\mathrm{~dB})$ \\
\hline & & & 0.8663 & 0.4663 & 0.7495 & 0.7495 & 0.7495 & \multirow[t]{3}{*}{63.7} \\
\hline & & & $R T 6$ & $R T 7$ & $R T 8$ & $R T 9$ & $R T 10$ & \\
\hline & & & 0.7495 & 0.006558 & 0.0941 & 0.006558 & 0.0941 & \\
\hline \multirow[t]{4}{*}{5} & \multirow[t]{4}{*}{0.96} & \multirow[t]{4}{*}{400} & $R T 1$ & $R T 2$ & $R T 3$ & $R T 4$ & $R T 5$ & STL $(\mathrm{dB})$ \\
\hline & & & 0.8785 & 0.4785 & 0.7677 & 0.7677 & 0.7677 & \multirow[t]{3}{*}{69.1} \\
\hline & & & $R T 6$ & $R T 7$ & $R T 8$ & $R T 9$ & $R T 10$ & \\
\hline & & & 0.7677 & 0.006718 & \begin{tabular}{|l|}
0.09623 \\
\end{tabular} & 0.006718 & 0.09623 & \\
\hline \multirow[t]{4}{*}{6} & \multirow[t]{4}{*}{0.96} & \multirow[t]{4}{*}{800} & $R T 1$ & $R T 2$ & $R T 3$ & $R T 4$ & $R T 5$ & $S T L(\mathrm{~dB})$ \\
\hline & & & 0.8829 & 0.4829 & 0.7744 & 0.7744 & 0.7744 & \multirow[t]{3}{*}{81.4} \\
\hline & & & $R T 6$ & $R T 7$ & $R T 8$ & $R T 9$ & $R T 10$ & \\
\hline & & & 0.7744 & 0.006776 & 0.09701 & 0.006776 & 0.09701 & \\
\hline \multirow[t]{4}{*}{7} & \multirow[t]{4}{*}{$\underline{0.96}$} & \multirow[t]{4}{*}{$\underline{1000}$} & $R T 1$ & $R T 2$ & $R T 3$ & $R T 4$ & $R T 5$ & $S T L(\mathrm{~dB})$ \\
\hline & & & $\underline{0.8748}$ & 0.4748 & $\underline{0.7622}$ & $\underline{0.7622}$ & 0.7622 & \multirow[t]{3}{*}{$\underline{85.2}$} \\
\hline & & & $R T 6$ & $R T 7$ & $R T 8$ & $R T 9$ & $R T 10$ & \\
\hline & & & $\underline{0.7622}$ & $\underline{0.006669}$ & 0.09559 & $\underline{0.006669}$ & $\underline{0.09559}$ & \\
\hline
\end{tabular}

where $k k$ is the cooling rate; moreover, to reach an initial transition probability of 0.5 , the initial temperature $\left(T_{o}\right)$ is selected as 0.2 [13]. The process is repeated until the predetermined number $\left(\right.$ iter $_{\max }$ ) of the outer loop is reached.

\section{RESULTS AND DISCUSSION}

\section{Results}

To achieve good optimization, two kinds of $S A$ parameters, including the cooling rate $(\mathrm{kk})$ and the number of iterations (Iter) are varied step by step during optimization. The optimization system is encoded by Fortran and run on an IBM PC - Pentium IV. Two results of optimization (one, pure tone noises used for $S A$ 's accuracy check; and the other, a broadband noise occurring in a fan room) are described below.

\section{1) Pure Tone Noise Optimization}

Seven sets of SA parameters are tested by varying the values of the SA parameters. The simulated results with respect to a pure tone of $250 \mathrm{~Hz}$ is summarized and shown in Table 2.

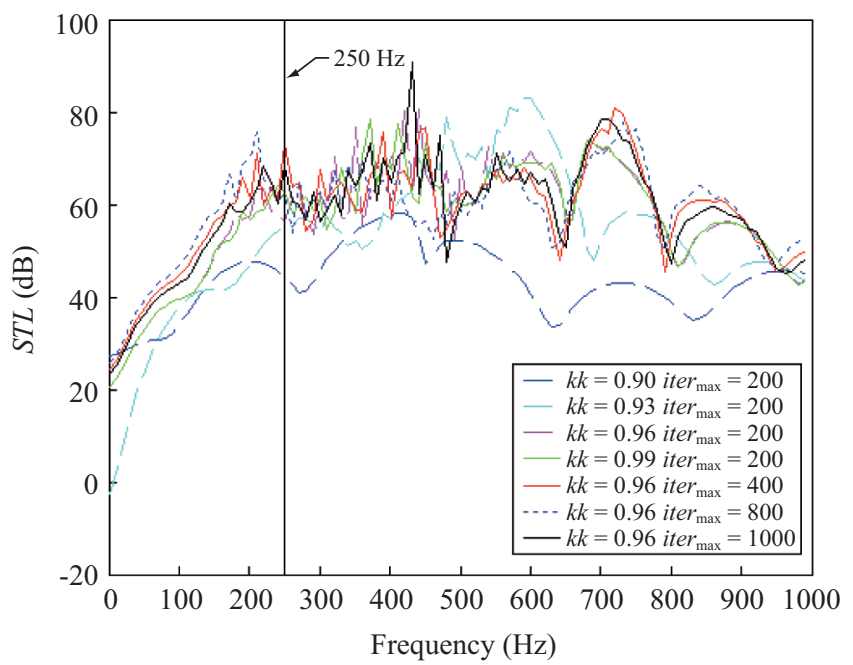

Fig. 11. $S T L$ with respect to frequency at various $k k$ and iter $_{\max }$ [target tone of $250 \mathrm{~Hz}$.

As indicated in Table 2, the optimal design data can be obtained from the last set of $S A$ parameters at $\left(k k\right.$, iter $\left.r_{\max }\right)=(0.96,1000)$. Using the optimal design in a theoretical calculation, the optimal $S T L$ curves with respect to various SA parameters are plotted and depicted in Fig. 11. As revealed in Fig. 11, the STLs are roughly maximized at the desired frequencies.

\section{2) Broadband Noise Optimization}

By using the above SA parameters, the muffler's optimal design data for one-chamber mufflers hybridized with perforated intruding tubes used to minimize the sound power level at the muffler's outlet is summarized in Table 3. As illustrated in Table 3, the resultant sound power levels with respect to three kinds of mufflers have been dramatically reduced from $122.4 \mathrm{~dB}(\mathrm{~A})$ to $80.1 \mathrm{~dB}(\mathrm{~A})$. Using this optimal design in a theoretical calculation, the optimal STL curves with respect to various SA parameters are plotted and compared with the original SWL depicted in Fig. 12.

To appreciate the acoustic performance, two kinds of low back-pressure mufflers (a one-chamber simple expansion muffler and a one-chamber muffler hybridized with non-perforated intruding tubes) are optimized. As indicated in Table 4, the resultant $S W L$ with respect to three mufflers (a one-chamber simple expansion muffler, a one-chamber muffler hybridized with perforated intruding tubes, and a one-chamber muffler hybridized with non-perforated intruding tubes) are $94.2 \mathrm{~dB}$, $80.1 \mathrm{~dB}$, and $109.4 \mathrm{~dB}$. Using this optimal design in a theoretical calculation, the optimal $S T L$ curves with respect to three kinds of optimized mufflers are plotted and compared with original $S W L$ shown in Fig. 13.

\section{Discussion}

To achieve a sufficient optimization, the selection of the appropriate SA parameters set is essential. As indicated in Table 2 , the best SA set at the targeted pure tone noise of $250 \mathrm{~Hz}$ has 
Table 3. Optimal $S W L$ for a one-chamber muffler with perforated intruding tubes at various $k k$ and iter $_{\text {max }}$ (broadband noise).

\begin{tabular}{|c|c|c|c|c|c|c|c|c|}
\hline \multirow{3}{*}{$\frac{\text { Item }}{1}$} & \multicolumn{2}{|c|}{ SA parameters } & \multirow{2}{*}{\multicolumn{6}{|c|}{ Results }} \\
\hline & \multirow{5}{*}{$\begin{array}{c}k k \\
0.90\end{array}$} & \multirow{5}{*}{$\begin{array}{c}\text { iter }_{\text {max }} \\
40\end{array}$} & & & & & & \\
\hline & & & $R T 1$ & $R T 2$ & $R T 3$ & $R T 4$ & $R T 5$ & \multirow{4}{*}{$\frac{S W L_{\mathrm{T}}(\mathrm{dB}}{98.3}$} \\
\hline & & & 0.8729 & 0.4729 & 0.7593 & 0.7593 & 0.7593 & \\
\hline & & & $R T 6$ & $R T 7$ & $R T 8$ & $R T 9$ & $R T 10$ & \\
\hline & & & 0.7593 & 0.006644 & 0.09525 & 0.006644 & 0.09525 & \\
\hline \multirow[t]{4}{*}{2} & \multirow[t]{4}{*}{0.93} & \multirow[t]{4}{*}{40} & $R T 1$ & $R T 2$ & $R T 3$ & $R T 4$ & $R T 5$ & $S W L_{\mathrm{T}}(\mathrm{dB})$ \\
\hline & & & 0.6361 & 0.2361 & 0.4041 & 0.4041 & 0.4041 & \multirow[t]{3}{*}{93.2} \\
\hline & & & $R T 6$ & $R T 7$ & $R T 8$ & $R T 9$ & $R T 10$ & \\
\hline & & & 0.4041 & 0.003536 & 0.05381 & 0.003536 & 0.05381 & \\
\hline \multirow[t]{4}{*}{3} & \multirow[t]{4}{*}{$\underline{0.96}$} & \multirow[t]{4}{*}{40} & $R T 1$ & $R T 2$ & $R T 3$ & $R T 4$ & $R T 5$ & $S W L_{\mathrm{T}}(\mathrm{dB})$ \\
\hline & & & 0.6043 & 0.2043 & 0.3564 & 0.3564 & 0.3564 & \multirow[t]{3}{*}{90.0} \\
\hline & & & $R T 6$ & $R T 7$ & $R T 8$ & $R T 9$ & $R T 10$ & \\
\hline & & & 0.3564 & 0.003119 & 0.04825 & \begin{tabular}{|l|l|} 
\\
\end{tabular} & 0.04825 & \\
\hline \multirow[t]{4}{*}{4} & \multirow[t]{4}{*}{0.99} & \multirow[t]{4}{*}{40} & $R T 1$ & $R T 2$ & $R T 3$ & $R T 4$ & $R T 5$ & $S W L_{\mathrm{T}}(\mathrm{dB})$ \\
\hline & & & 0.5617 & 0.1617 & 0.2926 & 0.2926 & 0.2926 & \multirow[t]{3}{*}{92.2} \\
\hline & & & $R T 6$ & $R T 7$ & $R T 8$ & $R T 9$ & $R T 10$ & \\
\hline & & & 0.2926 & \begin{tabular}{|l|}
0.00256 \\
\end{tabular} & 0.0408 & \begin{tabular}{|l|}
0.00256 \\
\end{tabular} & 0.0408 & \\
\hline \multirow[t]{4}{*}{5} & \multirow[t]{4}{*}{0.96} & \multirow[t]{4}{*}{80} & $R T 1$ & $R T 2$ & $R T 3$ & $R T 4$ & $R T 5$ & $S W L_{\mathrm{T}}(\mathrm{dB})$ \\
\hline & & & 0.5354 & 0.1354 & 0.2531 & 0.2531 & 0.2531 & \multirow[t]{3}{*}{88.2} \\
\hline & & & $R T 6$ & $R T 7$ & $R T 8$ & $R T 9$ & $R T 10$ & \\
\hline & & & 0.2531 & 0.002214 & 0.03619 & 0.002214 & 0.03619 & \\
\hline \multirow[t]{4}{*}{6} & \multirow[t]{4}{*}{0.96} & \multirow[t]{4}{*}{160} & $R T 1$ & $R T 2$ & $R T 3$ & $R T 4$ & $R T 5$ & $S W L_{\mathrm{T}}(\mathrm{dB})$ \\
\hline & & & 0.5485 & 0.1485 & 0.2727 & 0.2727 & 0.2727 & \multirow[t]{3}{*}{86.4} \\
\hline & & & $R T 6$ & $R T 7$ & $R T 8$ & $R T 9$ & $R T 10$ & \\
\hline & & & 0.2727 & 0.002386 & 0.03849 & 0.002386 & 0.03849 & \\
\hline \multirow[t]{4}{*}{7} & 0.96 & 320 & $R T 1$ & $R T 2$ & $R T 3$ & $R T 4$ & $R T 5$ & $S W L_{\mathrm{T}}(\mathrm{dB})$ \\
\hline & & & 0.8159 & 0.4159 & 0.6738 & 0.6738 & 0.6738 & 84.4 \\
\hline & & & RT6 & $R T 7$ & $R T 8$ & $R T 9$ & $R T 10$ & \\
\hline & & & 0.6738 & \begin{tabular}{|l|}
0.005896 \\
\end{tabular} & 0.08528 & 0.005896 & 0.08528 & \\
\hline 8 & $\underline{0.96}$ & $\underline{640}$ & $R T 1$ & $R T 2$ & $R T 3$ & $R T 4$ & $R T 5$ & $S W L_{\mathrm{T}}(\mathrm{dB})$ \\
\hline & & & $\underline{0.8101}$ & $\underline{0.4101}$ & $\underline{0.6651}$ & $\underline{0.6651}$ & $\underline{0.6651}$ & $\underline{80.1}$ \\
\hline & & & $R T 6$ & $R T 7$ & $R T 8$ & $R T 9$ & $R T 10$ & \\
\hline & & & 0.6651 & \begin{tabular}{|l|l|}
0.00582 \\
\end{tabular} & 0.08426 & \begin{tabular}{|l|}
0.00582 \\
\end{tabular} & 0.08426 & \\
\hline
\end{tabular}

been shown. The related $S T L$ curves with respect to various SA parameters are plotted in Fig. 11. Figure 11 reveals the predicted maximal value of the $S T L$ is located at the desired frequency. Therefore, using the SA optimization in finding a better design solution is reliable; moreover, in dealing with the broadband noise, the SA's solution shown in Table 3 and Fig. 12 can also provide the appropriate and sufficient sound reduction under space-constraint conditions. To investigate the acoustical performance among three kinds of low backpressure mufflers (a one-chamber simple expansion muffler, a one-chamber muffler hybridized with perforated intruding tubes, and a one-chamber muffler hybridized with non-perforated intruding tubes), the resultant $S W L_{\mathrm{T}}$ is shown in Table 4 and plotted in Fig. 13. It is obvious that the one-chamber muffler hybridized with perforated intruding tubes is superior to the other mufflers. As can be observed in Table 4, the overall sound transmission loss of the one-chamber muffler with perforated

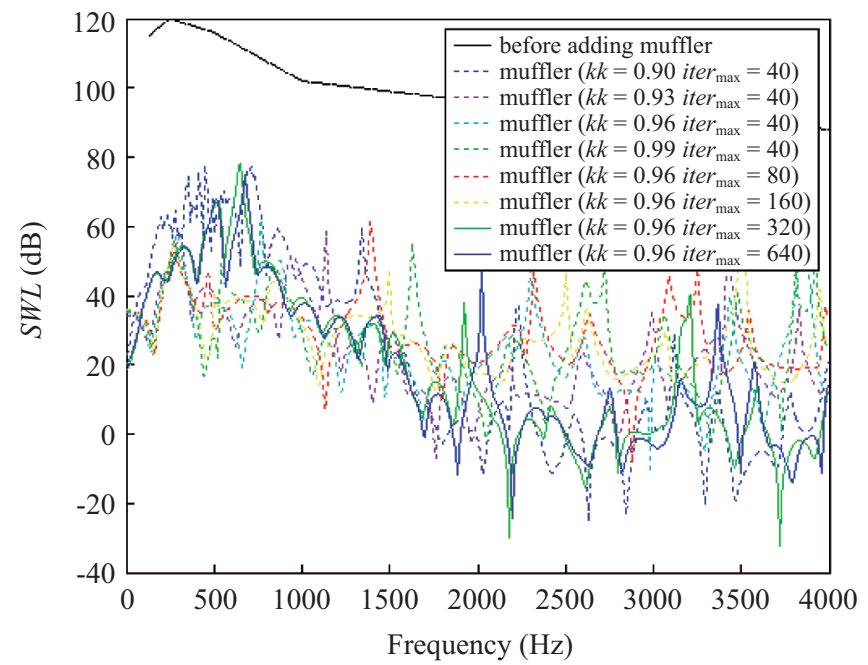

Fig. 12. Comparison of the optimal $S T L$ with respect to original noise level at $k k=0.96$ and iter $_{\max }=640$ [broadband noise].

Table 4. Comparison of acoustical performance with respect to three kinds of optimized mufflers within a same space-constrained situation (broadband noise).

\begin{tabular}{|c|c|c|c|c|c|c|c|}
\hline Item & Muffler Type & \multicolumn{6}{|c|}{ Results } \\
\hline \multirow[t]{2}{*}{1} & \multirow[t]{2}{*}{$\begin{array}{l}\text { one-chamber simple } \\
\text { expansion muffler }\end{array}$} & \multicolumn{2}{|c|}{$R T 1^{*}$} & \multicolumn{2}{|c|}{$R T 2^{*}$} & $R T 3^{*}$ & $S W L_{\mathrm{T}}$ \\
\hline & & \multicolumn{2}{|c|}{0.1163} & \multicolumn{2}{|c|}{0.2245} & 0.2245 & 94.2 \\
\hline \multirow[t]{4}{*}{2} & \multirow{4}{*}{$\begin{array}{c}\text { one-chamber muffler } \\
\text { equipped with } \\
\text { perforated intruding } \\
\text { tubes }\end{array}$} & $R T 1$ & $R T 2$ & $R T 3$ & $R T 4$ & $R T 5$ & $\begin{array}{c}S W L_{\mathrm{T}} \\
(\mathrm{dB})\end{array}$ \\
\hline & & 0.8101 & 0.4101 & 0.6651 & 0.6651 & 0.6651 & \multirow[t]{3}{*}{\begin{tabular}{|l}
80.1 \\
\end{tabular}} \\
\hline & & $R T 6$ & $R T 7$ & $R T 8$ & $R T 9$ & $R T 10$ & \\
\hline & & 0.6651 & 0.00582 & 0.08426 & 0.00582 & 0.08426 & \\
\hline \multirow[t]{4}{*}{3} & \multirow{4}{*}{$\begin{array}{c}\text { one-chamber muffler } \\
\text { equipped with } \\
\text { non-perforated } \\
\text { intruding tubes }\end{array}$} & \multicolumn{2}{|c|}{$R T 1^{* *}$} & \multicolumn{3}{|c|}{$R T 2^{* *}$} & $\begin{array}{c}S W L_{\mathrm{T}} \\
(\mathrm{dB})\end{array}$ \\
\hline & & \multicolumn{2}{|c|}{0.2926} & \multicolumn{3}{|c|}{0.2926} & \multirow[t]{3}{*}{109.4} \\
\hline & & \multicolumn{2}{|c|}{$R T 3^{* *}$} & \multicolumn{3}{|c|}{$R T 4^{* *}$} & \\
\hline & & \multicolumn{2}{|c|}{0.2926} & \multicolumn{3}{|c|}{0.2926} & \\
\hline
\end{tabular}

Note 1 (for one-chamber muffler equipped with non-perforated intruding tubes):

$L_{z}^{* *}=R T 1^{* * *} L_{o} ; L_{1}^{* *}=L_{5}^{* *}=\left(L_{0}-L_{z}^{* *}\right) / 2 ; L_{3}^{* *}=R T 2^{* *}{ }^{* *} L_{z}^{* *} ; L_{2}^{* *}=L_{4}^{* *}=\left(L_{z}^{* *}-L_{3}^{* *}\right) / 2 ; D_{1}^{* *}=$ $R T 3^{* * *} D_{o} ; D_{2}^{* *}=R T_{4}^{* * *} D_{o}$

Note 2 (for one-chamber simple expansion muffler):

$L_{2}{ }^{*}=R T 1^{*} * L_{o} ; L_{1}{ }^{*}=L_{3}{ }^{*}=\left(L_{o}-L_{2}{ }^{*}\right) / 2 ; D_{1}{ }^{*}=R T 2^{*} * D_{o} ; D_{2}{ }^{*}=R T 3^{*} * D_{o}$

intruding tubes reaches $43.9 \mathrm{~dB}$. However, the overall sound transmission loss of the one-chamber simple expansion muffler and the one-chamber muffler with non-perforated intruding tubes are $28 \mathrm{~dB}$ and $12.8 \mathrm{~dB}$.

\section{CONCLUSION}

It has been shown that one-chamber mufflers hybridized with perforated intruding tubes can be easily and efficiently optimized within a limited space by using a decoupling technique, a plane wave theory, a four-pole transfer matrix, and a SA optimizer. Two kinds of SA parameters $\left(k k\right.$, iter $\left.r_{\max }\right)$ play 


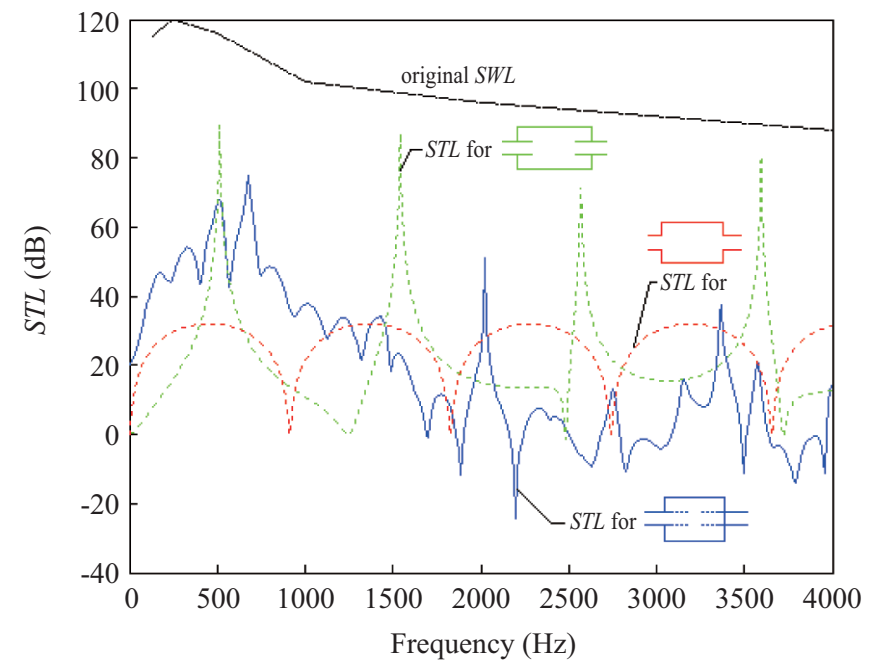

Fig. 13. Comparison of the optimal $S T L$ s of three kinds of mufflers with respect to original noise level [broadband noise].

essential roles in the solution's accuracy during SA optimization. As indicated in Fig. 11, the tuning ability established by adjusting design parameters of mufflers is reliable.

In addition, the appropriate acoustical performance curve of three kinds of low back-pressure mufflers (a one-chamber simple expansion muffler, a one-chamber muffler hybridized with perforated intruding tubes, and a one-chamber muffler hybridized with non-perforated intruding tubes) has been assessed. As indicated in Table 4, the resultant $S W L_{\mathrm{T}}$ with respect to these mufflers is $94.2 \mathrm{~dB}, 80.1 \mathrm{~dB}$, and $109.4 \mathrm{~dB}$. As shown in Table 4 and Fig. 13, it is quite obvious that the acoustical mechanism using perforated intruding tubes inside the muffler's cavity has the best acoustical performance compared to those with no tubes and non-perforated intruding tubes. Consequently, the approach used for the optimal design of the $S T L$ proposed in this study is quite efficient in dealing with the industrial venting noise within a space-constrained situation.

\section{ACKNOWLEDGMENTS}

The authors acknowledge the financial support of the National Science Council (NSC 97-2221-E-235-001, ROC).

\section{NOMENCLATURE}

This paper is constructed on the basis of the following notations:

$\begin{array}{ll}C: & \text { Boltzmann's factor } \\ C_{o}: & \text { sound speed }\left(\mathrm{m} \mathrm{s}^{-1}\right) \\ k_{1}, k_{2}: & \text { coefficients } \\ d h_{i}: & \text { the diameter of a perforated hole on the } \\ & \text { ith inner tube }(\mathrm{m}) \\ D_{\mathrm{i}}: & \text { diameter of the ith perforated tubes }(\mathrm{m}) \\ D_{\mathrm{o}}: & \text { diameter of the outer tube }(\mathrm{m})\end{array}$

$f:$
iter $_{\max }:$
$j:$
$k:$
$k k:$
$C_{1}, C_{2}, C_{3}, C_{4}:$
$C C_{1}, C C_{2}, C C_{3}, C C_{4}:$
$L_{C 1}, L_{C 2}:$
$L_{o}:$
$M:$
$O B J_{i}:$
$p:$
$\bar{p}_{i}:$
$p b(T):$
$Q:$
$S_{i}:$
$S T L:$
$S W L O:$

$S W L_{T}:$

$t_{\mathrm{i}}:$

$\mathrm{TS} 1_{\mathrm{ij}}, \mathrm{TS} 3_{\mathrm{ij}}$ :

$\mathrm{TPOE}_{\mathrm{ij}}$ :

$\mathrm{TPOC}_{\mathrm{ij}}$ :

$\mathrm{T}_{i j}^{*}:$

$T:$

$T_{o}$ :

$U:$

$u$ :

$\bar{u}_{i}$ :

$u$ :

$V_{1}$ :

$V_{2}$ :

$\rho_{o}:$

$\rho_{i}$ :

$\varsigma_{i}:$

$\eta_{i}$ : cyclic frequency $(\mathrm{Hz})$

maximum iteration

imaginary unit

wave number $\left(=\frac{\omega}{c_{o}}\right)$

cooling rate in $\mathrm{SA}$

coefficients in function $C_{i} e^{\beta_{i} x}$

coefficients in function $C C_{i} e^{\varepsilon_{i} x}$

lengths of perforate straight ducts (m)

total length of the muffler (m)

mean flow Mach number

objective function $(\mathrm{dB})$

acoustic pressure $(\mathrm{Pa})$

acoustic pressure at the ith node $(\mathrm{Pa})$

transition probability

volume flow rate of venting gas $\left(\mathrm{m}^{3} \mathrm{~s}^{-1}\right)$

section area at the ith node $\left(\mathrm{m}^{2}\right)$

sound transmission loss $(\mathrm{dB})$

unsilenced sound power level inside the muffler's inlet (dB)

overall sound power level inside the muffler's output (dB)

the thickness of the ith inner perforated tube (m)

components of four-pole transfer matrices for an acoustical mechanism with straight ducts

components of a four-pole transfer matrix for an acoustical mechanism with a perforated intruding inlet tube

components of a four-pole transfer matrix for an acoustical mechanism with a perforated intruding outlet tube

components of a four-pole transfer sys-

tem matrix

current temperature $\left({ }^{\circ} \mathrm{C}\right)$

initial temperature $\left({ }^{\circ} \mathrm{C}\right)$

potential energy

acoustic particle velocity $\left(\mathrm{m} \mathrm{s}^{-1}\right)$

acoustic particle velocity at the $i t h$ node $\left(\mathrm{m} \mathrm{s}^{-1}\right)$

acoustical particle velocity passing

through a perforated hole from the

ith node to the jth node $\left(\mathrm{m} \mathrm{s}^{-1}\right)$

mean flow velocity at the inner perforated tube $\left(\mathrm{m} \mathrm{s}^{-1}\right)$

mean flow velocity at the outer tube $\left(\mathrm{m} \mathrm{s}^{-1}\right)$

air density $\left(\mathrm{kg} \mathrm{m}^{-3}\right)$

acoustical density at the $i t h$ node

specific acoustical impedance of the ith inner perforated tube

the porosity of the ith inner perforated 
tube.

$\beta_{i}: \quad$ ith eigen value of $[\mathrm{N}]_{4 \times 4}$

$[\Omega]_{4 \times 4}$ : $\quad$ the model matrix formed by four sets of eigen vectors $\Omega_{4 \times 1}$ of $[\mathrm{N}]_{4 \times 4}$

\section{REFERENCES}

1. Chang, Y. C., Yeh, L. J., and Chiu, M. C., "GA optimization on singlechamber muffler hybridized with extended tube under space constraints," Archives of Acoustics, Vol. 29, No. 4, pp. 577-596 (2004).

2. Chang, Y. C., Yeh, L. J., and Chiu, M. C., "Shape optimization on constrained single-chamber muffler by using GA method and mathematical gradient method," International Journal of Acoustics and Vibration, Vol. 10, No. 1, pp. 17-25 (2005).

3. Chang, Y. C., Yeh, L. J., Chiu, M. C., and Lai, G. J., "Computer aided design on single expansion muffler with extended tube under space constraints," Tamkang Journal of Science and Engineering, Vol. 7, No. 3, pp. 171-181 (2004).

4. Davis, D. D., Stokes, J. M., and Moorse, L., "Theoretical and experimental investigation of mufflers with components on engine muffler design," NACA Report, pp. 1192 (1954).

5. Jayaraman, K. and Yam, K., "Decoupling approach to modeling perforated tube muffler component," Journal of the Acoustical Society of America, Vol. 69, No. 2, pp. 390-396 (1981).

6. Kim, Y. H., Choi, J. W., and Lim, B. D., "Acoustic characteristics of an expansion chamber with constant mass flow and steady temperature gradient (theory and numerical simulation)," Journal of Vibration and Acoustics, Vol. 112, pp. 460-467 (1990).

7. Kirkpatrick, S., Gelatt, C. D., and Vecchi, M. P., "Optimization by simulated annealing," Science, Vol. 220, No. 4598, pp. 671-680 (1983).

8. Magrab, E. B., Environmental Noise Control, John Wiley \& Sons, New York (1975).

9. Metropolis, A., Rosenbluth, W., Rosenbluth, M. N., Teller, H., and Teller, E., "Equation of static calculations by fast computing machines," Journal of Chemical Physics, Vol. 21, No. 6, pp. 1087-1092 (1953).

10. Munjal, M. L., Acoustics of Ducts and Mufflers with Application to Exhaust and Ventilation System Design, John Wiley \& Sons, New York
(1987).

11. Munjal, M. L., "Plane wave analysis of side inlet/outlet chamber mufflers with mean flow," Applied Acoustics, Vol. 52, No. 2, pp. 165-175 (1997).

12. Munjal, M. L., Rao, K. N., and Sahasrabudhe, A. D., "Aeroacoustic analysis of perforated muffler components," Journal of Sound and Vibration, Vol. 114, No. 2, pp. 173-88 (1987).

13. Nolle, L., Armstrong, D. A., Hopgood, A. A., and Ware, J. A., "Simulated annealing and genetic algorithms applied to finishing mill optimization for hot rolling of wide steel strip," International of Knowledge-Based Intelligent Engineering System, Vol. 6, No. 2, pp. 104-111 (2002).

14. Peat, K. S., "A numerical decoupling analysis of perforated pipe silencer elements," Journal of Sound and Vibration, Vol. 123, No. 2, pp. 199-212 (1988).

15. Prasad, M. G., "A note on acoustic plane waves in a uniform pipe with mean flow," Journal of Sound and Vibration, Vol. 95, No. 2, pp. 284-290 (1984).

16. Rao, K. N. and Munjal, M. L., "A generalized decoupling method for analyzing perforated element mufflers," Nelson Acoustics Conference, Madison (1984).

17. Sullivan, J. W., "A method of modeling perforated tube muffler components I: Theory," Journal of the Acoustical Society of America, Vol. 66, pp $772-778$ (1979).

18. Sullivan, J. W., "A method of modeling perforated tube muffler components II: Applications," Journal of the Acoustical Society of America, Vol. 66, pp. 779-788 (1979).

19. Sullivan, J. W. and Crocker, M. J., "Analysis of concentric tube resonators having unpartitioned cavities," Journal of the Acoustical Society of America, Vol. 64, pp. 207-215 (1978).

20. Thawani, P. T. and Jayaraman, K., "Modeling and applications of straight-through resonators," Journal of the Acoustical Society of America, Vol. 73, No. 4, pp. 1387-1389 (1983).

21. Wang, C. N., "A numerical scheme for the analysis of perforated intruding tube muffler components," Applied Acoustics, Vol. 44, pp. 275-286 (1995).

22. Yeh, L. J., Chang, Y. C., Chiu, M. C., and Lai, G. J., "GA optimization on multi-segments muffler under space constraints," Applied Acoustics, Vol. 65, No. 5, pp. 521-543 (2004). 